\title{
Finite-element approximation of a nonlinear degenerate parabolic system describing bacterial pattern formation
}

\author{
JOHN W. BARRETT ${ }^{\dagger}$ AND ROBERT NÜRNBERG \\ Department of Mathematics, Imperial College, London SW7 2BZ, UK
}

[Received 6 September 2001 and in revised form 27 February 2002]

We consider a fully practical finite-element approximation of the following nonlinear degenerate parabolic system

$$
\begin{aligned}
\frac{\partial u}{\partial t}-c \Delta u & =-f(u) v \quad \text { in } \Omega_{T}:=\Omega \times(0, T), \quad \Omega \subset \mathbf{R}^{d}, d \leqslant 2 ; \\
\frac{\partial v}{\partial t}-\nabla .(b(u, v) \nabla v) & =\theta f(u) v \quad \text { in } \Omega_{T}
\end{aligned}
$$

subject to no flux boundary conditions, and non-negative initial data $u^{0}$ and $v^{0}$ on $u$ and $v$. Here we assume that $c>0, \theta \geqslant 0$ and that $f(r) \geqslant f(0)=0$ is Lipschitz continuous and monotonically increasing for $r \in\left[0, \sup _{x \in \Omega} u^{0}(x)\right]$. Throughout the paper we restrict ourselves to the model degenerate case $b(u, v):=\sigma u v$, where $\sigma>0$. The above models the spatiotemporal evolution of a bacterium on a thin film of nutrient, where $u$ is the nutrient concentration and $v$ is the bacterial cell density. In addition to showing stability bounds for our approximation, we prove convergence and hence existence of a solution to this nonlinear degenerate parabolic system. Finally, some numerical experiments in one and two space dimensions are presented.

Keywords: bacterial pattern formation; nonlinear degenerate parabolic systems; finite element approximation.

\section{Introduction}

We consider the initial boundary value problem

(P) Find functions $u, v: \Omega \times[0, T] \rightarrow \mathbf{R}_{\geqslant 0}$ such that

$$
\begin{aligned}
\frac{\partial u}{\partial t}-c \Delta u & =-g(u, v) & & \text { in } \Omega_{T}:=\Omega \times(0, T), \\
\frac{\partial v}{\partial t}-\nabla \cdot(b(u, v) \nabla v) & =\theta g(u, v) & & \text { in } \Omega_{T} \\
u(x, 0)=u^{0}(x) \geqslant 0, \quad v(x, 0) & =v^{0}(x) \geqslant 0 & & \forall x \in \Omega, \\
\frac{\partial u}{\partial v}=b(u, v) \frac{\partial v}{\partial v} & =0 & & \text { on } \partial \Omega \times(0, T) ;
\end{aligned}
$$

where $\Omega$ is a bounded domain in $\mathbf{R}^{d}, d \leqslant 2$, with a Lipschitz boundary $\partial \Omega, v$ is normal to $\partial \Omega$ and $T>0$ is a fixed positive time. A system of the type (P) was proposed in [7] of nutrient, where $u$ is the nutrient concentration and $v$ is the bacterial cell density. The diffusion coefficients of the

† Corresponding author. Email: j.barrett@ic.ac.uk 
nutrient and bacterial cells are $c>0$ and $b(\cdot, \cdot) \geqslant 0$, respectively. Throughout the paper, we will restrict ourselves to the model case:

$$
b(r, s):=\sigma r s, \quad \text { where } \sigma>0 \text {; }
$$

that is, $b(r, s)$ degenerates if either $r$ or $s=0$. The term $g(\cdot, \cdot) \geqslant 0$ represents the consumption rate of nutrient by the cells; while $\theta g(\cdot, \cdot)$ is the growth rate of bacteria, where $\theta \geqslant 0$ is the conversion rate of consumed nutrient to bacterial growth. We assume throughout that $g(\cdot, \cdot)$ is monotonically increasing in both arguments. More precisely we assume that $g(\cdot, \cdot) \in C(\mathbf{R} \geqslant 0, \mathbf{R} \geqslant 0)$ satisfies

$$
\begin{array}{ll}
g\left(r_{2}, s\right) \geqslant g\left(r_{1}, s\right) \geqslant g(0, s)=0 & \forall r_{2} \geqslant r_{1} \geqslant 0, s \geqslant 0 ; \\
g\left(r, s_{2}\right) \geqslant g\left(r, s_{1}\right) \geqslant 0 & \forall r \geqslant 0, s_{2} \geqslant s_{1} \geqslant 0 .
\end{array}
$$

The degeneracy in both arguments of $b(\cdot, \cdot)$ makes the analysis of this system particularly difficult. In fact we are unaware of any existence proof or any numerical analysis work on such a system, even though there is a great deal of interest in the degenerate system $(\mathrm{P})$ in the mathematical/theoretical biology community, see e.g. $[5,8]$ and the references therein. Related models describing bacterial pattern formation are also discussed in these review papers. In this paper we propose and analyse a fully practical finite-element approximation of $(\mathrm{P})$ and as a consequence we prove existence of a weak solution. For the stability bounds on this approximation, we will restrict ourselves to

$$
g(r, s):=f(r) s, \text { where } f(\cdot) \in C\left(\mathbf{R}_{\geqslant 0}\right) \text { and } f\left(r_{2}\right) \geqslant f\left(r_{1}\right) \geqslant f(0)=0 \quad \forall r_{2} \geqslant r_{1} \geqslant 0 \text {; }
$$

that is, $g(r, s)$ has linear growth in $s$. For our main convergence result, we will further assume that $f(r)$ is Lipschitz continuous for $r \in\left[0, \sup _{x \in \Omega} u^{0}(x)\right]$, see (3.18) below. In any case, all of our assumptions on $b(\cdot, \cdot)$ and $g(\cdot, \cdot)$ include the models proposed in [7]: (1.2) with either MichaelisMenten kinetics, $g(r, s):=\rho r s /(1+\gamma r)$, or its bilinear approximation, $g(r, s):=\rho r s$; where $\rho, \gamma>0$.

In analysing our approximation of (P) we will adapt some of the techniques used by [4]; where they prove convergence, in two space dimensions, of a finite-element approximation of the following phase field model for diffusion-induced grain motion: find functions $u: \Omega_{T} \rightarrow[-1,1]$ and $v$ : $\Omega_{T} \rightarrow \mathbf{R}$ such that

$$
\begin{array}{ll}
\frac{\partial u}{\partial t}-c \Delta u-\theta u+\rho v+\partial I_{[-1,1]}(u) \ni 0 & \text { in } \Omega_{T} \\
\frac{\partial v}{\partial t}=\nabla .(D(u) \nabla v) & \text { in } \Omega_{T}
\end{array}
$$

where $\partial I_{[-1,1]}$ is the subdifferential of $I_{[-1,1]}$, the indicator function of the set $[-1,1], D(r):=$ $\sigma\left(1-r^{2}\right)$ and $\sigma, c, \theta, \rho>0$. The system $(1.5 \mathrm{a}, \mathrm{b})$ is supplemented by initial and flux boundary conditions for $u$ and $v$. Here (1.5a) is a double obstacle Allen-Cahn equation with a forcing obtained from the solution of the degenerate diffusion equation (1.5b). One can clearly see similarities between $(1.1 \mathrm{a}, \mathrm{b})$ and $(1.5 \mathrm{a}, \mathrm{b})$. However, the degeneracy in $(1.1 \mathrm{~b})$ is harder to deal with as $b(\cdot, \cdot)$ degenerates with respect to both arguments. A double degeneracy of the type occurring in $(\mathrm{P})$ has been considered, for example, in [2]; where they prove convergence, in one space dimension, of a finite element approximation of the following degenerate Allen-Cahn/Cahn-Hilliard system: find 
functions $u, v, w, z: \Omega_{T} \rightarrow \mathbf{R}$ such that

$$
\begin{aligned}
\frac{\partial u}{\partial t} & =\nabla \cdot(D(u, v) \nabla w) & & \text { in } \Omega_{T}, \\
\rho \frac{\partial v}{\partial t} & =-D(u, v) z & & \text { in } \Omega_{T}, \\
w & =-c \Delta u+\theta[\phi(u+v)+\phi(u-v)]-\alpha u & & \text { in } \Omega_{T}, \\
z & =-c \Delta v+\theta[\phi(u+v)-\phi(u-v)]-\beta v & & \text { in } \Omega_{T} ;
\end{aligned}
$$

where $\phi(r):=\ln r-\ln (1-r), D(r, s):=\sigma r(1-r)\left(1-4 s^{2}\right)$ and $\sigma, c, \theta, \rho, \alpha, \beta>0$. The system $(1.6 \mathrm{a}-\mathrm{d})$ is supplemented by initial conditions for $u, v$ and no flux boundary conditions for $u, v, w$. Substituting $(1.6 \mathrm{c}, \mathrm{d})$ into $(1.6 \mathrm{a}, \mathrm{b})$, respectively, the above system consists of a degenerate fourth-order equation coupled to a degenerate second-order equation. In some ways (1.6a-d) is more difficult than the degenerate system $(\mathrm{P})$ as (a) both equations are degenerate, (b) $\phi$ is a singular nonlinearity forcing $(u \pm v)(x, t) \in(0,1)$. However, the structure of the system (1.6a-d) allows one to prove the following uniform bounds:

$$
\|U\|_{L^{\infty}\left(0, T ; H^{1}(\Omega)\right)}+\|V\|_{L^{\infty}\left(0, T ; H^{1}(\Omega)\right)}+\left\|\frac{\partial U}{\partial t}\right\|_{L^{2}\left(0, T ;\left(H^{1}(\Omega)\right)^{\prime}\right)}+\left\|\frac{\partial V}{\partial t}\right\|_{L^{2}\left(\Omega_{T}\right)} \leqslant C
$$

for a solution $\{U, V\}_{h}$ of an appropriate finite-element approximation, where $h$ is the discretization parameter. In one space dimension, on noting the compact embedding $H^{1}(\Omega) \hookrightarrow C(\bar{\Omega})$ and $(1.14 \mathrm{~b})$ below, the bounds (1.7) give rise to the uniform subsequence convergence $U \rightarrow u, V \rightarrow v$ on $\Omega_{T}$ as $h \rightarrow 0$. This uniform convergence plays an essential role and hence the restriction to one space dimension, $d=1$, in [2]. Unfortunately, the bounds on $V$ in (1.7) do not hold for an approximation of $(\mathrm{P})$. Hence the approach in [2] is not appropriate for $(\mathrm{P})$, even for $d=1$. As stated above, in this paper we adapt the techniques in [4] to prove convergence of our finite-element approximation of (P) for $d \leqslant 2$. To achieve this, we use a special discretization of $b(\cdot, \cdot)$ in our finite element approximation that enables us to prove a key a priori bound. This approach is based on an idea introduced in [14] and [6] for the thin-film equation: Find $u: \Omega_{T} \rightarrow \mathbf{R}$ such that

$$
\frac{\partial u}{\partial t}+\nabla \cdot\left(|u|^{p} \nabla \Delta u\right)=0 \quad \text { in } \Omega_{T},
$$

where $p>0$. We now motivate this technique for (P). On assuming (1.2) and (1.4), it is easy to establish $L^{\infty}\left(\Omega_{T}\right)$ bounds on $u$, $v$ for $(\mathrm{P})$; see Section 2 for such bounds on the finite-element approximation of $(\mathrm{P})$. Then testing a weak formulation of $(1.1 \mathrm{~b})$ with $v$ yields that

$$
\sigma \int_{\Omega_{T}} u v|\nabla v|^{2} \mathrm{~d} x \mathrm{~d} t \leqslant \frac{1}{2}\left\|v^{0}\right\|_{L^{2}(\Omega)}^{2}+\theta \int_{\Omega_{T}} f(u) v^{2} \mathrm{~d} x \mathrm{~d} t \leqslant C .
$$

The above bound only controls $v|\nabla v|^{2}$, and hence $\left|\nabla\left(v^{2}\right)\right|^{2}$, where $u>0$. However, if we define a function $\mathcal{L}(\cdot)$ such that

$$
\eta \nabla\left[\mathcal{L}^{\prime}(\eta)\right]=\nabla \eta \quad \Longrightarrow \quad \mathcal{L}^{\prime \prime}(s)=s^{-1} \Rightarrow \mathcal{L}(s)=s(\ln s-1)+1 ;
$$

then testing $(1.1 \mathrm{~b})$ with $\mathcal{L}^{\prime}(v)$ yields that

$$
\sigma \int_{\Omega_{T}} u|\nabla v|^{2} \mathrm{~d} x \mathrm{~d} t \leqslant \int_{\Omega} \mathcal{L}\left(v^{0}\right) \mathrm{d} x+\theta \int_{\Omega_{T}} f(u) \mathcal{L}^{\prime}(v) v \mathrm{~d} x \mathrm{~d} t \leqslant C .
$$


This bound controls $|\nabla v|^{2}$ where $u>0$. The discrete analogue of (1.11) will play a key role in the convergence analysis in Section 3. To obtain such a bound we require a discrete analogue of (1.10) and hence the special approximation of $b(\cdot, \cdot)$.

This paper is organized as follows. In Section 2 we formulate a fully practical finite-element approximation of problem (P) and derive stability bounds. In Section 3 we prove convergence of this approximation to a weak solution of $(\mathrm{P})$, in space dimensions $d \leqslant 2$, and hence we prove existence of a solution to $(\mathrm{P})$. In Section 4 we state an iterative scheme for solving the nonlinear discrete system at each time level and present some numerical computations in both one and two space dimensions.

\section{Notation and auxiliary results}

We adopt the standard notation for Sobolev spaces, denoting the norm of $W^{m, q}(\Omega)$ ( $m \in \mathbf{N}, q \in$ $[1, \infty])$ by $\|\cdot\|_{m, q}$ and the semi-norm by $|\cdot|_{m, q}$. For $q=2, W^{m, 2}(\Omega)$ will be denoted by $H^{m}(\Omega)$ with the associated norm and semi-norm written as, respectively, $\|\cdot\|_{m}$ and $|\cdot|_{m}$. We introduce also $\left(W^{m, q}(\Omega)\right)^{\prime}$ and $\left(H^{m}(\Omega)\right)^{\prime}$ the dual spaces of $W^{m, q}(\Omega)$ and $H^{m}(\Omega)$, respectively, and denote the norm of $\left(H^{m}(\Omega)\right)^{\prime}$ by $\|\cdot\|_{-m}$. Throughout, $(\cdot, \cdot)$ will denote the standard $L^{2}$ inner product over $\Omega$. In addition, we define

$$
f \eta:=\frac{1}{\underline{m}(\Omega)}(\eta, 1) \quad \forall \eta \in L^{1}(\Omega), \quad \text { where } \quad \underline{m}(\Omega) \text { is the measure of } \Omega .
$$

For later purposes, we recall the following well known Sobolev interpolation result, see e.g. [1]: Let $q \in[1, \infty], m \geqslant 1$,

$$
r \in \begin{cases}{[q, \infty]} & \text { if } m-\frac{d}{q}>0, \\ {[q, \infty)} & \text { if } m-\frac{d}{q}=0, \\ {\left[q,-\frac{d}{m-(d / q)}\right]} & \text { if } m-\frac{d}{q}<0,\end{cases}
$$

and $\mu=\frac{d}{m}\left(\frac{1}{q}-\frac{1}{r}\right)$. Then there is a constant $C$ depending only on $\Omega, q, r, m$ such that for all $z \in W^{m, q}(\Omega)$ the inequality

$$
|z|_{0, r} \leqslant C|z|_{0, q}^{1-\mu}\|z\|_{m, q}^{\mu}
$$

holds. We recall also the following compactness results. Let $X, Y$ and $Z$ be Banach spaces with a compact embedding $X \hookrightarrow Y$ and a continuous embedding $Y \hookrightarrow Z$. Then the embeddings

$$
\begin{aligned}
& \quad\left\{\eta \in L^{2}(0, T ; X): \frac{\partial \eta}{\partial t} \in L^{2}(0, T ; Z)\right\} \hookrightarrow L^{2}(0, T ; Y) \\
& \text { and } \quad\left\{\eta \in L^{\infty}(0, T ; X): \frac{\partial \eta}{\partial t} \in L^{2}(0, T ; Z)\right\} \hookrightarrow C([0, T] ; Y)
\end{aligned}
$$

are compact, see [13]. We note also for future reference Young's inequality

$$
r s \leqslant \frac{\gamma}{2} r^{2}+\frac{1}{2 \gamma} s^{2} \quad \forall r, s \in \mathbf{R}, \gamma \in \mathbf{R}_{>0} ;
$$

and the elementary identity

$$
2 r(r-s)=\left(r^{2}-s^{2}\right)+(r-s)^{2} \quad \forall r, s \in \mathbf{R} .
$$


Throughout, $C$ denotes a generic constant independent of $h, \tau$ and $\varepsilon$, the mesh and temporal discretization parameters and the regularization parameter. In addition $C\left(a_{1}, \ldots, a_{I}\right)$ denotes a constant depending on the arguments $\left\{a_{i}\right\}_{i=1}^{I}$.

\section{Finite-element approximation}

We consider the finite-element approximation of $(\mathrm{P})$ at first under the following assumptions on the mesh:

(A) Let $\Omega$ be a polygonal domain if $d=2$. Let $\left\{\mathcal{T}^{h}\right\}_{h>0}$ be a quasi-uniform family of partitionings of $\Omega$ into disjoint open simplices $\kappa$ with $h_{\kappa}:=\operatorname{diam}(\kappa)$ and $h:=\max _{\kappa \in \mathcal{T}^{h}} h_{\kappa}$, so that $\bar{\Omega}=$ $\cup_{\kappa \in \mathcal{T} h} \bar{\kappa}$. In addition, it is assumed for $d=2$ that all simplices $\kappa \in \mathcal{T}^{h}$ are right-angled.

We note that the quasi-uniformity assumption can be avoided at the expense of a mild constraint on the minimum time step, see Remark 3.1. Furthermore, we note that the right-angled simplices assumption is not a severe constraint, as there exist adaptive finite-element codes that satisfy this requirement, see e.g. [12].

Associated with $\mathcal{T}^{h}$ is the finite-element space

$$
S^{h}:=\left\{\chi \in C(\bar{\Omega}):\left.\chi\right|_{\kappa} \text { is linear } \forall \kappa \in \mathcal{T}^{h}\right\} \subset H^{1}(\Omega) .
$$

We introduce also

$$
K^{h}:=\left\{\chi \in S^{h}: \chi \geqslant 0 \text { in } \Omega\right\} \subset K:=\left\{\eta \in H^{1}(\Omega): \eta \geqslant 0 \text { a.e. in } \Omega\right\} .
$$

Let $J$ be the set of nodes of $\mathcal{T}^{h}$ and $\left\{p_{j}\right\}_{j \in J}$ the coordinates of these nodes. Let $\mathcal{J}:=\# J$ and $\left\{\chi_{j}\right\}_{j \in J}$ be the standard basis functions for $S^{h}$; that is $\chi_{j} \in K^{h}$ and $\chi_{j}\left(p_{i}\right)=\delta_{i j}$ for all $i, j \in J$. The right-angle constraint on the partitioning is required for our approximation of $b(\cdot, \cdot)$, see $(2.6 \mathrm{a}, \mathrm{b})$, but one consequence is that

$$
\int_{\kappa} \frac{\partial \chi_{j}}{\partial x_{i}} \frac{\partial \chi_{k}}{\partial x_{i}} \mathrm{~d} x \leqslant 0 \quad j \neq k, \quad i=1, \ldots, d, \quad \forall \kappa \in \mathcal{T}^{h} .
$$

We introduce $\pi^{h}: C(\bar{\Omega}) \rightarrow S^{h}$, the interpolation operator, such that $\pi^{h} \eta\left(p_{j}\right)=\eta\left(p_{j}\right)$ for all $j \in J$. A discrete inner product on $C(\bar{\Omega})$ is then defined by

$$
\left(\eta_{1}, \eta_{2}\right)^{h}:=\int_{\Omega} \pi^{h}\left(\eta_{1}(x) \eta_{2}(x)\right) \mathrm{d} x \equiv \sum_{j \in J} m_{j} \eta_{1}\left(p_{j}\right) \eta_{2}\left(p_{j}\right),
$$

where $m_{j}:=\left(1, \chi_{j}\right)>0$. We introduce the $L^{2}$ projection $Q^{h}: L^{2}(\Omega) \rightarrow S^{h}$ defined by

$$
\left(Q^{h} \eta, \chi\right)^{h}=(\eta, \chi) \quad \forall \chi \in S^{h} .
$$

For computational purposes, we replace $\mathcal{L} \in C^{\infty}\left(\mathbf{R}_{>0}\right)$, see (1.10), for any $\varepsilon \in(0,1)$ by the regularized function $\mathcal{L}_{\varepsilon}: \mathbf{R} \rightarrow \mathbf{R}_{\geqslant 0}$ such that

$$
\mathcal{L}_{\varepsilon}(s):= \begin{cases}\frac{s^{2}-\varepsilon^{2}}{2 \varepsilon}+(\ln \varepsilon-1) s+1 & s \leqslant \varepsilon \\ s(\ln s-1)+1 & \varepsilon \leqslant s .\end{cases}
$$


Hence $\mathcal{L}_{\varepsilon} \in C^{2,1}(\mathbf{R})$ with the first two derivatives of $\mathcal{L}_{\varepsilon}$ given by

$$
\mathcal{L}_{\varepsilon}^{\prime}(s):=\left\{\begin{array}{ll}
\varepsilon^{-1} s+\ln \varepsilon-1 & s \leqslant \varepsilon \\
\ln s & \varepsilon \leqslant s
\end{array} \quad \text { and } \quad \mathcal{L}_{\varepsilon}^{\prime \prime}(s):= \begin{cases}\varepsilon^{-1} & s \leqslant \varepsilon \\
s^{-1} & \varepsilon \leqslant s,\end{cases}\right.
$$

respectively. Similarly to the approach in [14] and [6], we introduce $\Lambda_{\varepsilon}: S^{h} \rightarrow\left[L^{\infty}(\Omega)\right]^{d \times d}$ such that for all $z^{h} \in S^{h}$ and a.e. in $\Omega$

$$
\begin{aligned}
& \Lambda_{\varepsilon}\left(z^{h}\right) \text { is symmetric and positive semidefinite, } \\
& \qquad \Lambda_{\varepsilon}\left(z^{h}\right) \nabla \pi^{h}\left[\mathcal{L}_{\varepsilon}^{\prime}\left(z^{h}\right)\right]=\nabla z^{h}
\end{aligned}
$$

that is, the discrete analogue of (1.10). Firstly, we give the construction of $\Lambda_{\varepsilon}$ in the simple case when $d=1$. Given $z^{h} \in S^{h}$ and $\kappa \in \mathcal{T}^{h}$ having vertices $p_{j}$ and $p_{k}$, we set

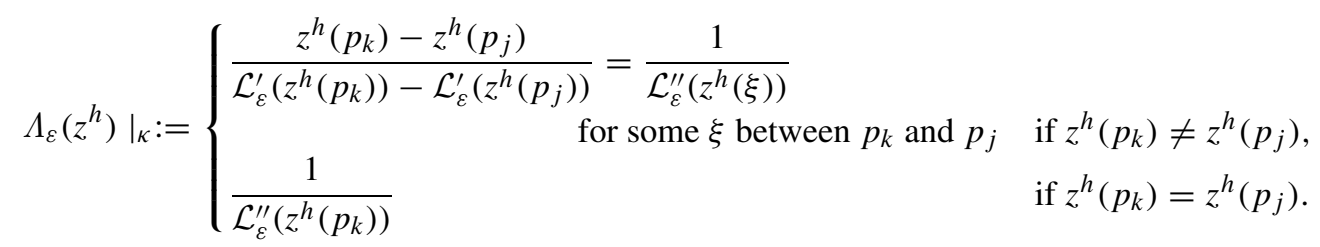

Clearly the piecewise constant construction in (2.7) satisfies the conditions (2.6a,b). Following [6] we extend the above construction to $d=2$. Let $\left\{e_{i}\right\}_{i=1}^{d}$ be the orthonormal vectors in $\mathbf{R}^{d}$, such that the $j$ th component of $e_{i}$ is $\delta_{i j}, i, j=1, \ldots, d$. Given non-zero constants $\alpha_{i}, i=1, \ldots, d$; let $\widehat{\kappa}\left(\left\{\alpha_{i}\right\}_{i=1}^{d}\right)$ be the reference open simplex in $\mathbf{R}^{d}$ with vertices $\left\{\widehat{p}_{i}\right\}_{i=0}^{d}$, where $\widehat{p}_{0}$ is the origin and $\widehat{p}_{i}=\alpha_{i} e_{i}, i=1, \ldots, d$. Given a $\kappa \in \mathcal{T}^{h}$ with vertices $\left\{p_{j_{i}}\right\}_{i=0}^{d}$, such that $p_{j_{0}}$ is the right-angled vertex, then there exists a rotation matrix $R_{\kappa}$ and non-zero constants $\left\{\alpha_{i}\right\}_{i=1}^{d}$ such that the mapping $\mathcal{R}_{\kappa}: \widehat{x} \in \mathbf{R}^{d} \rightarrow p_{j_{0}}+R_{\kappa} \widehat{x} \in \mathbf{R}^{d}$ maps the vertex $\widehat{p}_{i}$ to $p_{j_{i}}, i=0, \ldots, d$, and hence $\widehat{\kappa} \equiv \widehat{\kappa}\left(\left\{\alpha_{i}\right\}_{i=1}^{d}\right)$ to $\kappa$. For any $z^{h} \in S^{h}$, we then set

$$
\left.\Lambda_{\varepsilon}\left(z^{h}\right)\right|_{\kappa}:=\left.R_{\kappa} \widehat{\Lambda}_{\varepsilon}\left(z^{h}\right)\right|_{\widehat{\kappa}} R_{\kappa}^{T},
$$

where $\widehat{z}^{h}(\widehat{x}) \equiv z^{h}\left(\mathcal{R}_{\kappa} \widehat{x}\right)$ for all $\widehat{x} \in \bar{\kappa}$ and $\left.\widehat{\Lambda}_{\varepsilon}\left(\bar{z}^{h}\right)\right|_{\widehat{\kappa}}$ is the $d \times d$ diagonal matrix with diagonal entries, $k=1, \ldots, d$,

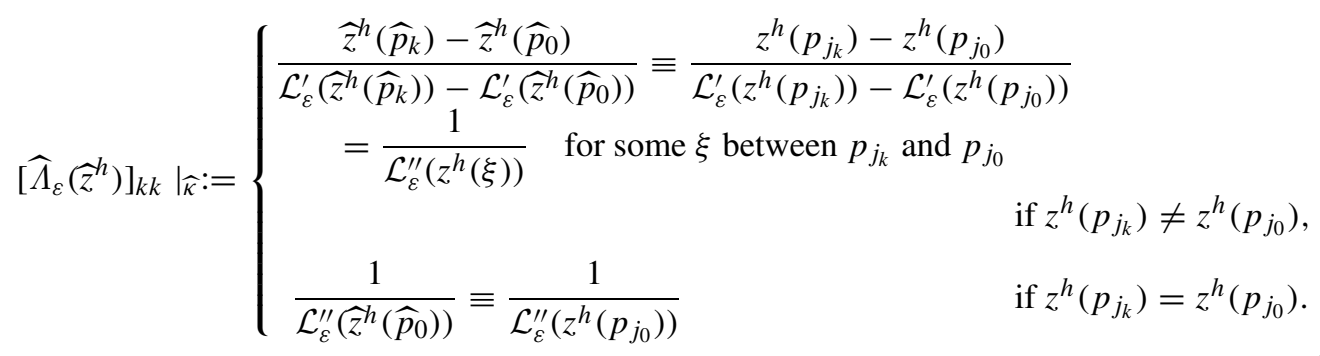

As $R_{\kappa}^{T} \equiv R_{\kappa}^{-1}, \nabla z^{h} \equiv R_{\kappa} \widehat{\nabla} \widehat{z}^{h}$, where $x \equiv\left(x_{1}, \cdots, x_{d}\right)^{T}, \nabla \equiv\left(\frac{\partial}{\partial x_{1}}, \cdots, \frac{\partial}{\partial x_{d}}\right)^{T}, \widehat{x} \equiv\left(\widehat{x}_{1}, \cdots, \widehat{x}_{d}\right)^{T}$ and $\widehat{\nabla} \equiv\left(\frac{\partial}{\partial \widehat{x}_{1}}, \cdots, \frac{\partial}{\partial \widehat{x}_{d}}\right)^{T}$, it easily follows that $\Lambda_{\varepsilon}\left(z^{h}\right)$ constructed in (2.8) and (2.9) satisfies (2.6a,b). It is this construction that requires the right-angle constraint on the partitioning $\mathcal{T}^{h}$. 
For the purposes of the analysis it is convenient to extend the domain of $g(\cdot, \cdot)$ to $\mathbf{R} \times \mathbf{R}$ by setting

$$
g(r, s)=0 \quad \forall r \leqslant 0, \forall s \in \mathbf{R} \quad \text { and } \quad g(r, s)=g(r, 0) \quad \forall s \leqslant 0, \forall r \in \mathbf{R} .
$$

In addition to $\mathcal{T}^{h}$, let $0 \equiv t_{0}<t_{1}<\cdots<t_{N-1}<t_{N} \equiv T$ be a partitioning of [0,T] into possibly variable time steps $\tau_{n}:=t_{n}-t_{n-1}, n=1, \ldots, N$. We set $\tau:=\max _{n=1, \ldots, N} \tau_{n}$. For any given $\varepsilon \in(0,1)$, we then consider the following fully practical finite-element approximation of $(\mathrm{P})$ with $b(\cdot, \cdot)$ as in (1.2):

$\left(\mathbf{P}_{\varepsilon}^{h, \tau}\right)$ For $n \geqslant 1$, find $\left\{U_{\varepsilon}^{n}, V_{\varepsilon}^{n}\right\} \in S^{h} \times S^{h}$ such that

$$
\begin{array}{ll}
\left(\frac{U_{\varepsilon}^{n}-U_{\varepsilon}^{n-1}}{\tau_{n}}, \chi\right)^{h}+c\left(\nabla U_{\varepsilon}^{n}, \nabla \chi\right)+\left(g\left(U_{\varepsilon}^{n}, V_{\varepsilon}^{n-1}\right), \chi\right)^{h}=0 & \forall \chi \in S^{h}, \\
\left(\frac{V_{\varepsilon}^{n}-V_{\varepsilon}^{n-1}}{\tau_{n}}, \chi\right)^{h}+\sigma\left(U_{\varepsilon}^{n} \Lambda_{\varepsilon}\left(V_{\varepsilon}^{n}\right) \nabla V_{\varepsilon}^{n}, \nabla \chi\right)=\theta\left(g\left(U_{\varepsilon}^{n}, V_{\varepsilon}^{n-1}\right), \chi\right)^{h} & \forall \chi \in S^{h},
\end{array}
$$

where $U_{\varepsilon}^{0}, V_{\varepsilon}^{0} \in K^{h}$ are approximations of $u^{0}, v^{0} \geqslant 0$, respectively, e.g. $U_{\varepsilon}^{0} \equiv \pi^{h} u^{0}$ or $Q^{h} u^{0}$ and similarly $V_{\varepsilon}^{0}$. In fact, it is a simple matter to deduce that a solution of $\left(\mathrm{P}_{\varepsilon}^{h, \tau}\right)$ is such that $\left\{U_{\varepsilon}^{n}, V_{\varepsilon}^{n}\right\} \in K^{h} \times K^{h}, n=1, \ldots, N$, see $(2.39$ a,b) below.

Below we recall some well known results concerning $S^{h}$ for $m=0$ or 1:

$$
\begin{array}{rlrl}
|\chi|_{1, \kappa} \leqslant C h_{\kappa}^{-1}|\chi|_{0, \kappa} & & \forall \chi \in S^{h}, \quad \forall \kappa \in \mathcal{T}^{h} ; \\
\left|\left(I-\pi^{h}\right) \eta\right|_{m} & \leqslant C h^{2-m}|\eta|_{2} & & \forall \eta \in H^{2}(\Omega) ; \\
\left|\left(I-\pi^{h}\right) \eta\right|_{m} & \leqslant C h^{1-m}|\eta|_{1} & & \forall \eta \in H^{1}(\Omega), \quad \text { if } d=1 ; \\
\left|\left(I-\pi^{h}\right) f(\chi)\right|_{0} & \leqslant C h\left|\pi^{h}[f(\chi)]\right|_{1} & & \forall \chi \in K^{h} ;
\end{array}
$$

and

$$
\begin{array}{cc}
|\chi|_{0} \leqslant|\chi|_{h}:=\left[(\chi, \chi)^{h}\right]^{\frac{1}{2}} \leqslant(d+2)^{\frac{1}{2}}|\chi|_{0} \quad & \forall \chi \in S^{h} ; \\
\left|\left(z^{h}, \chi\right)-\left(z^{h}, \chi\right)^{h}\right| \leqslant\left|\left(I-\pi^{h}\right)\left(z^{h} \chi\right)\right|_{0,1} \leqslant C h^{1+m}\left|z^{h}\right|_{m}|\chi|_{1} & \forall z^{h}, \chi \in S^{h} .
\end{array}
$$

It follows from (2.3) that

$$
\left(Q^{h} \eta\right)\left(x_{j}\right) \equiv \frac{\left(\eta, \chi_{j}\right)}{\left(1, \chi_{j}\right)} \quad \forall j \in J \quad \Longrightarrow \quad\left\|Q^{h} \eta\right\|_{0, \infty} \leqslant\|\eta\|_{0, \infty} \quad \forall \eta \in L^{\infty}(\Omega) .
$$

Finally, as we have a quasi-uniform family of partitionings, it holds for $m=0$ or 1 that

$$
\left|\left(I-Q^{h}\right) \eta\right|_{m} \leqslant C h^{1-m}\|\eta\|_{1} \quad \forall \eta \in H^{1}(\Omega) .
$$

We require the following results concerning $\Lambda_{\varepsilon}(\cdot)$.

LEMma 2.1 Let the assumptions (A) hold and let $\|\cdot\|$ denote the spectral norm on $\mathbf{R}^{d \times d}$. Then for any given $\varepsilon \in(0,1)$ the function $\Lambda_{\varepsilon}: S^{h} \rightarrow\left[L^{\infty}(\Omega)\right]^{d \times d}$ satisfies

$$
\varepsilon \xi^{T} \xi \leqslant \xi^{T} \Lambda_{\varepsilon}\left(z^{h}\right) \xi \leqslant \max \left(\varepsilon,\left\|z^{h}\right\|_{0, \infty}\right) \xi^{T} \xi \quad \forall \xi \in \mathbf{R}^{d} \quad \forall z^{h} \in S^{h}
$$


and is continuous. In particular it holds for all $z_{1}^{h}, z_{2}^{h} \in S^{h}, \kappa \in \mathcal{T}^{h}$ that

$$
\begin{aligned}
& \left\|\left.\left[\Lambda_{\varepsilon}\left(z_{1}^{h}\right)-\Lambda_{\varepsilon}\left(z_{2}^{h}\right)\right]\right|_{\kappa}\right\|=\left\|\left.\left[\widehat{\Lambda}_{\varepsilon}\left(\widehat{z}_{1}^{h}\right)-\widehat{\Lambda}_{\varepsilon}\left(z_{2}^{h}\right)\right]\right|_{\widehat{\kappa}}\right\| \\
& \quad \leqslant \varepsilon^{-1} \max \left(\varepsilon,\left\|z_{1}^{h}\right\|_{0, \infty},\left\|z_{2}^{h}\right\|_{0, \infty}\right) \max _{k=1, \ldots, d}\left[\left|z_{1}^{h}\left(p_{j_{k}}\right)-z_{2}^{h}\left(p_{j_{k}}\right)\right|+\left|z_{1}^{h}\left(p_{j_{0}}\right)-z_{2}^{h}\left(p_{j_{0}}\right)\right|\right],
\end{aligned}
$$

where we have adopted the notation (2.8) and (2.9).

Proof. (2.20) follows immediately from (2.8), (2.9) and (2.5). The equality in (2.21) follows immediately from (2.8). Adopting the notation (2.8) and (2.9) we have that

$$
\left\|\left.\left[\widehat{\Lambda}_{\varepsilon}\left(\hat{z}_{1}^{h}\right)-\widehat{\Lambda}_{\varepsilon}\left(\bar{z}_{2}^{h}\right)\right]\right|_{\widehat{\kappa}}\right\|=\max _{k=1, \ldots, d}\left|\left[\left[\widehat{\Lambda}_{\varepsilon}\left(\hat{z}_{1}^{h}\right)\right]_{k k}-\left[\widehat{\Lambda}_{\varepsilon}\left(\bar{z}_{2}^{h}\right)\right]_{k k}\right]\right| \widehat{\kappa} \mid
$$

and

$$
\begin{aligned}
\mid\left[\left[\widehat{\Lambda}_{\varepsilon}\left(\hat{z}_{1}^{h}\right)\right]_{k k}-\right. & {\left.\left[\widehat{\Lambda}_{\varepsilon}\left(\bar{z}_{2}^{h}\right)\right]_{k k}\right]\left.\right|_{\widehat{\kappa}} \mid } \\
& \leqslant\left|\left[\left[\widehat{\Lambda}_{\varepsilon}\left(\bar{z}_{1}^{h}\right)\right]_{k k}-\left[\widehat{\Lambda}_{\varepsilon}\left(\widehat{z}_{1,2}^{h}\right)\right]_{k k}\right]\right| \widehat{\kappa}|+|\left[\left[\widehat{\Lambda}_{\varepsilon}\left(\widehat{z}_{1,2}^{h}\right)\right]_{k k}-\left[\widehat{\Lambda}_{\varepsilon}\left(\bar{z}_{2}^{h}\right)\right]_{k k}\right]|\widehat{\kappa}| \\
& =\left|\frac{1}{\mathcal{L}_{\varepsilon}^{\prime \prime}\left(\mu_{1}\right)}-\frac{1}{\mathcal{L}_{\varepsilon}^{\prime \prime}(\zeta)}\right|+\left|\frac{1}{\mathcal{L}_{\varepsilon}^{\prime \prime}(\zeta)}-\frac{1}{\mathcal{L}_{\varepsilon}^{\prime \prime}\left(\mu_{2}\right)}\right|,
\end{aligned}
$$

where $z_{1,2}^{h} \in S^{h}$ with $z_{1,2}^{h}\left(p_{j_{0}}\right)=z_{2}^{h}\left(p_{j_{0}}\right)$ and $z_{1,2}^{h}\left(p_{j_{k}}\right)=z_{1}^{h}\left(p_{j_{k}}\right), \mu_{i}$ lies between $z_{i}^{h}\left(p_{j_{0}}\right)$ and $z_{i}^{h}\left(p_{j_{k}}\right), i=1,2$, and $\zeta$ lies between $z_{1}^{h}\left(p_{j_{k}}\right)$ and $z_{2}^{h}\left(p_{j_{0}}\right)$.

We now show that

$$
\left|\frac{1}{\mathcal{L}_{\varepsilon}^{\prime \prime}\left(\mu_{1}\right)}-\frac{1}{\mathcal{L}_{\varepsilon}^{\prime \prime}(\zeta)}\right| \leqslant \varepsilon^{-1} \max \left(\varepsilon,\left|z_{1}^{h}\left(p_{j_{0}}\right)\right|,\left|z_{2}^{h}\left(p_{j_{0}}\right)\right|\right)\left|z_{1}^{h}\left(p_{j_{0}}\right)-z_{2}^{h}\left(p_{j_{0}}\right)\right|,
$$

for $z_{1}^{h}\left(p_{j_{0}}\right) \neq z_{2}^{h}\left(p_{j_{0}}\right)$. If $z_{1}^{h}\left(p_{j_{0}}\right)=z_{2}^{h}\left(p_{j_{0}}\right)$, then $\zeta=\mu_{1}$ and (2.23) is trivially true. Otherwise let $\gamma$, lying between $z_{1}^{h}\left(p_{j_{0}}\right)$ and $z_{2}^{h}\left(p_{j_{0}}\right)$, be such that

$$
\frac{1}{\mathcal{L}_{\varepsilon}^{\prime \prime}(\gamma)}=\frac{z_{2}^{h}\left(p_{j_{0}}\right)-z_{1}^{h}\left(p_{j_{0}}\right)}{\mathcal{L}_{\varepsilon}^{\prime}\left(z_{2}^{h}\left(p_{j_{0}}\right)\right)-\mathcal{L}_{\varepsilon}^{\prime}\left(z_{1}^{h}\left(p_{j_{0}}\right)\right)}
$$

and so

$$
\left[z_{2}^{h}\left(p_{j_{0}}\right)-z_{1}^{h}\left(p_{j_{0}}\right)\right] \mathcal{L}_{\varepsilon}^{\prime \prime}(\gamma)=\left[z_{2}^{h}\left(p_{j_{0}}\right)-z_{1}^{h}\left(p_{j_{k}}\right)\right] \mathcal{L}_{\varepsilon}^{\prime \prime}(\zeta)+\left[z_{1}^{h}\left(p_{j_{k}}\right)-z_{1}^{h}\left(p_{j_{0}}\right)\right] \mathcal{L}_{\varepsilon}^{\prime \prime}\left(\mu_{1}\right) .
$$

It holds that either

$$
\begin{array}{rlrl} 
& \text { (i) } \quad & \left|z_{1}^{h}\left(p_{j_{k}}\right)-z_{1}^{h}\left(p_{j_{0}}\right)\right|+\left|z_{1}^{h}\left(p_{j_{k}}\right)-z_{2}^{h}\left(p_{j_{0}}\right)\right| & =\left|z_{1}^{h}\left(p_{j_{0}}\right)-z_{2}^{h}\left(p_{j_{0}}\right)\right| \\
\text { or } \quad(i i) & \left|z_{1}^{h}\left(p_{j_{0}}\right)-z_{2}^{h}\left(p_{j_{0}}\right)\right|+\left|z_{1}^{h}\left(p_{j_{k}}\right)-z_{2}^{h}\left(p_{j_{0}}\right)\right| & =\left|z_{1}^{h}\left(p_{j_{k}}\right)-z_{1}^{h}\left(p_{j_{0}}\right)\right| \\
\text { or } \quad(i i i) & & \left|z_{1}^{h}\left(p_{j_{k}}\right)-z_{1}^{h}\left(p_{j_{0}}\right)\right|+\left|z_{1}^{h}\left(p_{j_{0}}\right)-z_{2}^{h}\left(p_{j_{0}}\right)\right| & =\left|z_{1}^{h}\left(p_{j_{k}}\right)-z_{2}^{h}\left(p_{j_{0}}\right)\right| .
\end{array}
$$

In case $(i)$ we have on noting $\mathcal{L}_{\varepsilon}^{\prime \prime}(s)=[\max (s, \varepsilon)]^{-1}$ that

$$
\left|\frac{1}{\mathcal{L}_{\varepsilon}^{\prime \prime}\left(\mu_{1}\right)}-\frac{1}{\mathcal{L}_{\varepsilon}^{\prime \prime}(\zeta)}\right| \leqslant\left|\mu_{1}-\zeta\right| \leqslant\left|z_{1}^{h}\left(p_{j_{0}}\right)-z_{2}^{h}\left(p_{j_{0}}\right)\right| .
$$


For case $(i i)$ it follows from (2.24) that

$$
\left(z_{1}^{h}\left(p_{j_{k}}\right)-z_{1}^{h}\left(p_{j_{0}}\right)\right)\left[\frac{1}{\mathcal{L}_{\varepsilon}^{\prime \prime}(\zeta)}-\frac{1}{\mathcal{L}_{\varepsilon}^{\prime \prime}\left(\mu_{1}\right)}\right]=\left(z_{2}^{h}\left(p_{j_{0}}\right)-z_{1}^{h}\left(p_{j_{0}}\right)\right) \frac{\mathcal{L}_{\varepsilon}^{\prime \prime}(\gamma)}{\mathcal{L}_{\varepsilon}^{\prime \prime}\left(\mu_{1}\right)}\left[\frac{1}{\mathcal{L}_{\varepsilon}^{\prime \prime}(\zeta)}-\frac{1}{\mathcal{L}_{\varepsilon}^{\prime \prime}(\gamma)}\right],
$$

and hence we have that

$$
\begin{aligned}
\left|\frac{1}{\mathcal{L}_{\varepsilon}^{\prime \prime}\left(\mu_{1}\right)}-\frac{1}{\mathcal{L}_{\varepsilon}^{\prime \prime}(\zeta)}\right| & \leqslant \frac{\mathcal{L}_{\varepsilon}^{\prime \prime}(\gamma)}{\mathcal{L}_{\varepsilon}^{\prime \prime}\left(\mu_{1}\right)} \frac{|\zeta-\gamma|}{\left|z_{1}^{h}\left(p_{j_{k}}\right)-z_{1}^{h}\left(p_{j_{0}}\right)\right|}\left|z_{1}^{h}\left(p_{j_{0}}\right)-z_{2}^{h}\left(p_{j_{0}}\right)\right| \\
& \leqslant \varepsilon^{-1} \max \left(\varepsilon,\left|z_{1}^{h}\left(p_{j_{0}}\right)\right|,\left|z_{2}^{h}\left(p_{j_{0}}\right)\right|\right)\left|z_{1}^{h}\left(p_{j_{0}}\right)-z_{2}^{h}\left(p_{j_{0}}\right)\right| .
\end{aligned}
$$

For case $(i i i)$ we proceed similarly to end up with

$$
\begin{aligned}
\left|\frac{1}{\mathcal{L}_{\varepsilon}^{\prime \prime}\left(\mu_{1}\right)}-\frac{1}{\mathcal{L}_{\varepsilon}^{\prime \prime}(\zeta)}\right| & \leqslant \frac{\mathcal{L}_{\varepsilon}^{\prime \prime}(\gamma)}{\mathcal{L}_{\varepsilon}^{\prime \prime}(\zeta)} \frac{\left|\mu_{1}-\gamma\right|}{\left|z_{1}^{h}\left(p_{j_{k}}\right)-z_{2}^{h}\left(p_{j_{0}}\right)\right|}\left|z_{1}^{h}\left(p_{j_{0}}\right)-z_{2}^{h}\left(p_{j_{0}}\right)\right| \\
& \leqslant \varepsilon^{-1} \max \left(\varepsilon,\left|z_{1}^{h}\left(p_{j_{0}}\right)\right|,\left|z_{2}^{h}\left(p_{j_{0}}\right)\right|\right)\left|z_{1}^{h}\left(p_{j_{0}}\right)-z_{2}^{h}\left(p_{j_{0}}\right)\right| .
\end{aligned}
$$

Hence, combining (2.25a-c)-(2.28), we have shown the desired result (2.23). In a similar way we find that

$$
\left|\frac{1}{\mathcal{L}_{\varepsilon}^{\prime \prime}\left(\mu_{2}\right)}-\frac{1}{\mathcal{L}_{\varepsilon}^{\prime \prime}(\zeta)}\right| \leqslant \varepsilon^{-1} \max \left(\varepsilon,\left|z_{1}^{h}\left(p_{j_{k}}\right)\right|,\left|z_{2}^{h}\left(p_{j_{k}}\right)\right|\right)\left|z_{1}^{h}\left(p_{j_{k}}\right)-z_{2}^{h}\left(p_{j_{k}}\right)\right| .
$$

Combining (2.22a,b), (2.23) and (2.29) yields the desired result (2.21).

REMARK 2.1 The bound (2.21) is not pessimistic. For example, consider $d=1$, $z_{2}^{h}\left(p_{j_{0}}\right)=\varepsilon, z_{1}^{h}\left(p_{j_{1}}\right)=z_{2}^{h}\left(p_{j_{1}}\right)=1$ and $z_{1}^{h}\left(p_{j_{0}}\right)=\varepsilon+\gamma$ with $\gamma \in(0,1-\varepsilon)$. Then

$$
\begin{aligned}
{\left.\left[\Lambda_{\varepsilon}\left(z_{1}^{h}\right)-\Lambda_{\varepsilon}\left(z_{2}^{h}\right)\right]\right|_{\left(p_{j_{0}}, p_{j_{1}}\right)} } & =\frac{1-\varepsilon}{\ln \varepsilon}-\frac{1-(\varepsilon+\gamma)}{\ln (\varepsilon+\gamma)}=\frac{(1-\varepsilon) \ln \left(1+\varepsilon^{-1} \gamma\right)+\gamma \ln \varepsilon}{\ln (\varepsilon+\gamma) \ln \varepsilon} \\
& \sim(\ln \varepsilon)^{-2} \varepsilon^{-1} \gamma \quad \text { for } \gamma \ll \varepsilon \ll 1 .
\end{aligned}
$$

For any $\varepsilon \in(0,1)$, let $\lambda_{\varepsilon} \in C^{0,1}(\mathbf{R})$ be such that

$$
\begin{aligned}
& \lambda_{\varepsilon}(s):=[s-\varepsilon]_{+}+\varepsilon \quad \forall s \in \mathbf{R}, \quad \text { where } \quad[s]_{+}:=\max \{s, 0\}, \\
& \lambda_{\varepsilon}(s)-s \in[0, \varepsilon] \quad \forall s \in \mathbf{R}_{\geqslant 0} .
\end{aligned}
$$

LEMMA 2.2 Let the assumptions (A) hold. Then for any given $\varepsilon \in(0,1)$ the function $\Lambda_{\varepsilon}: S^{h} \rightarrow$ $\left[L^{\infty}(\Omega)\right]^{d \times d}$ is such that for all $z^{h} \in S^{h}$ and $\kappa \in \mathcal{T}^{h}$

$$
\max _{x \in \kappa}\left\|\left\{\Lambda_{\varepsilon}\left(z^{h}\right)-\pi^{h}\left[\lambda_{\varepsilon}\left(z^{h}\right)\right] \mathcal{I}\right\}(x)\right\| \leqslant h_{\kappa}\left|\nabla \pi^{h}\left[\lambda_{\varepsilon}\left(z^{h}\right)\right]\right|_{\kappa} \mid,
$$

where $\mathcal{I}$ is the $d \times d$ identity matrix.

Proof. Adopting the notation of (2.8), we have from (2.9) and (2.5) that

$$
\begin{aligned}
\max _{x \in \kappa}\left\|\left\{\Lambda_{\varepsilon}\left(z^{h}\right)-\pi^{h}\left[\lambda_{\varepsilon}\left(z^{h}\right)\right] \mathcal{I}\right\}(x)\right\|^{2} & =\max _{\widehat{x} \in \widehat{\kappa}}\left\|\left\{\widehat{\Lambda}_{\varepsilon}\left(\widehat{z}^{h}\right)-\widehat{\pi}^{h}\left[\lambda_{\varepsilon}\left(\hat{z}^{h}\right)\right] \mathcal{I}\right\}(\widehat{x})\right\|^{2} \\
& =\max _{\widehat{x} \in \widehat{\kappa}}\left\{\max _{k=1, \ldots, d}\left|\left[\widehat{\Lambda}_{\varepsilon}\left(\widehat{z}^{h}\right)\right]_{k k}-\widehat{\pi}^{h}\left[\lambda_{\varepsilon}\left(\widehat{z}^{h}\right)\right](\widehat{x})\right|^{2}\right\} \\
& \leqslant\left. h_{\kappa}^{2}\left|\widehat{\nabla} \widehat{\pi}^{h}\left[\lambda_{\varepsilon}\left(\widehat{z}^{h}\right)\right]\right|_{\widehat{\kappa}}\right|^{2}=\left.h_{\kappa}^{2}\left|\nabla \pi^{h}\left[\lambda_{\varepsilon}\left(z^{h}\right)\right]\right|_{\kappa}\right|^{2},
\end{aligned}
$$


where we have noted that $\left[\widehat{\Lambda}_{\varepsilon}\left(\widehat{z}^{h}\right)\right]_{k k}=\widehat{\pi}^{h}\left[\lambda_{\varepsilon}\left(\widehat{z}^{h}\right)\right]\left(\widehat{\xi}^{(k)}\right) \equiv \pi^{h}\left[\lambda_{\varepsilon}\left(z^{h}\right)\right]\left(\xi^{(k)}\right)$ with $\xi^{(k)} \equiv \mathcal{R}_{\kappa} \widehat{\xi}^{(k)} \in$ $\bar{\kappa}$ for some point $\widehat{\xi}^{(k)} \in \bar{\kappa}$. Hence we obtain the desired result (2.31).

In order to derive upper and lower bounds on $\left\{U_{\varepsilon}^{n}, V_{\varepsilon}^{n}\right\}_{n=1}^{N}$ we formulate the following scalar problems.

$\left(\mathbf{P}_{M}^{\tau}\right)$ For $n \geqslant 1$, let $\left\{\mathcal{U}_{M}^{n}, \mathcal{V}_{M}^{n}\right\} \in \mathbf{R} \geqslant 0 \times \mathbf{R}_{\geqslant 0}$ be such that

$$
\begin{aligned}
& \frac{\mathcal{U}_{M}^{n}-\mathcal{U}_{M}^{n-1}}{\tau_{n}}+g\left(\mathcal{U}_{M}^{n}, \mathcal{V}_{m}^{n-1}\right)=0 \\
& \frac{\mathcal{V}_{M}^{n}-\mathcal{V}_{M}^{n-1}}{\tau_{n}}=\theta g\left(\mathcal{U}_{M}^{n}, \mathcal{V}_{M}^{n-1}\right)
\end{aligned}
$$

$\left(\mathbf{P}_{m}^{\tau}\right)$ For $n \geqslant 1$, let $\left\{\mathcal{U}_{m}^{n}, \mathcal{V}_{m}^{n}\right\} \in \mathbf{R}_{\geqslant 0} \times \mathbf{R}_{\geqslant 0}$ be such that

$$
\begin{aligned}
& \frac{\mathcal{U}_{m}^{n}-\mathcal{U}_{m}^{n-1}}{\tau_{n}}+g\left(\mathcal{U}_{m}^{n}, \mathcal{V}_{M}^{n-1}\right)=0, \\
& \frac{\mathcal{V}_{m}^{n}-\mathcal{V}_{m}^{n-1}}{\tau_{n}}=\theta g\left(\mathcal{U}_{m}^{n}, \mathcal{V}_{m}^{n-1}\right),
\end{aligned}
$$

where $\mathcal{U}_{M}^{0}, \mathcal{U}_{m}^{0}, \mathcal{V}_{M}^{0}, \mathcal{V}_{m}^{0} \in \mathbf{R} \geqslant 0$ are such that for all $h>0$ and for all $\varepsilon \in(0,1)$

$$
\mathcal{U}_{M}^{0} \geqslant U_{\varepsilon}^{0}(x) \geqslant \mathcal{U}_{m}^{0} \quad \text { and } \quad \mathcal{V}_{M}^{0} \geqslant V_{\varepsilon}^{0}(x) \geqslant \mathcal{V}_{m}^{0} \quad \forall x \in \bar{\Omega} .
$$

LEMMA 2.3 Let the assumptions (1.3) on $g(\cdot, \cdot)$ hold. Let $\mathcal{U}_{M}^{0}, \mathcal{U}_{m}^{0}, \mathcal{V}_{M}^{0}, \mathcal{V}_{m}^{0}$ satisfy (2.34). Then for all time partitions $\left\{\tau_{n}\right\}_{n=1}^{N}$, there exists a unique solution $\left\{\mathcal{U}_{m}^{n}, \mathcal{V}_{m}^{n}, \mathcal{U}_{M}^{n}, \mathcal{V}_{M}^{n}\right\}_{n=1}^{N}$ to $\left(\mathrm{P}_{m}^{\tau}\right)$ and $\left(\mathrm{P}_{M}^{\tau}\right)$, respectively. Furthermore, it holds for all $n \geqslant 1$ that

$$
\begin{array}{lll}
\mathcal{U}_{M}^{n-1} \geqslant \mathcal{U}_{M}^{n} \geqslant \mathcal{U}_{m}^{n} & \text { and } & \mathcal{U}_{m}^{n-1} \geqslant \mathcal{U}_{m}^{n} \geqslant 0, \\
\mathcal{V}_{M}^{n} \geqslant \mathcal{V}_{M}^{n-1} & \text { and } & \mathcal{V}_{M}^{n} \geqslant \mathcal{V}_{m}^{n} \geqslant \mathcal{V}_{m}^{n-1} \geqslant 0 .
\end{array}
$$

Proof. We proceed by induction. Let

$$
\mathcal{U}_{M}^{n-1} \geqslant \mathcal{U}_{m}^{n-1} \geqslant 0 \quad \text { and } \quad \mathcal{V}_{M}^{n-1} \geqslant \mathcal{V}_{m}^{n-1} \geqslant 0,
$$

which is true for $n=1$ on noting (2.34). Existence and uniqueness of $\mathcal{U}_{M}^{n}, \mathcal{V}_{M}^{n}, \mathcal{U}_{m}^{n}, \mathcal{V}_{m}^{n}$ solving $(2.32 \mathrm{a}, \mathrm{b})$ and $(2.33 \mathrm{a}, \mathrm{b})$ follow immediately from our assumptions $(1.3)$ on $g(\cdot, \cdot)$. Moreover, it follows from (2.36), (2.32a), (2.33a) and (1.3) that

$$
\begin{aligned}
\mathcal{U}_{M}^{n}+\tau_{n} g\left(\mathcal{U}_{M}^{n}, \mathcal{V}_{m}^{n-1}\right)=\mathcal{U}_{M}^{n-1} \geqslant \mathcal{U}_{m}^{n-1} & =\mathcal{U}_{m}^{n}+\tau_{n} g\left(\mathcal{U}_{m}^{n}, \mathcal{V}_{M}^{n-1}\right) \\
& \geqslant \mathcal{U}_{m}^{n}+\tau_{n} g\left(\mathcal{U}_{m}^{n}, \mathcal{V}_{m}^{n-1}\right)
\end{aligned}
$$

and hence the desired result (2.35a). It follows from (2.36), (2.32b), (2.33b), (2.35a) and (1.3) that

$$
\mathcal{V}_{M}^{n}=\mathcal{V}_{M}^{n-1}+\tau_{n} \theta g\left(\mathcal{U}_{M}^{n}, \mathcal{V}_{M}^{n-1}\right) \geqslant \mathcal{V}_{m}^{n-1}+\tau_{n} \theta g\left(\mathcal{U}_{m}^{n}, \mathcal{V}_{m}^{n-1}\right)=\mathcal{V}_{m}^{n},
$$

and hence the desired result (2.35b). Therefore by induction the results $(2.35 \mathrm{a}, \mathrm{b})$ hold for all $n \geqslant 1$. 
THEOREM 2.1 Let the assumptions (A) hold, $g(\cdot, \cdot)$ satisfy (1.3), and $U_{\varepsilon}^{n-1}, V_{\varepsilon}^{n-1} \in K^{h}$. Then for all $\varepsilon \in(0,1)$ and for all $h, \tau_{n}>0$ there exists a solution $\left\{U_{\varepsilon}^{n}, V_{\varepsilon}^{n}\right\} \in K^{h} \times K^{h}$ to the $n$-th step of $\left(\mathrm{P}_{\varepsilon}^{h, \tau}\right)$. Furthermore $U_{\varepsilon}^{n}$ is unique.

Hence for all $\varepsilon \in(0,1), h>0$ and for all time partitions $\left\{\tau_{n}\right\}_{n=1}^{N}$, there exists a solution $\left\{U_{\varepsilon}^{n}, V_{\varepsilon}^{n}\right\}_{n=1}^{N}$ of $\left(\mathrm{P}_{\varepsilon}^{h, \tau}\right)$. In addition, if $\mathcal{U}_{M}^{0}, \mathcal{U}_{m}^{0}, \mathcal{V}_{M}^{0}, \mathcal{V}_{m}^{0}$ satisfy (2.34), then it follows that for all $n \geqslant 0$

$$
\begin{array}{ll}
\mathcal{U}_{M}^{0} \geqslant \mathcal{U}_{M}^{n} \geqslant U_{\varepsilon}^{n}(x) \geqslant \mathcal{U}_{m}^{n} \geqslant 0 & \forall x \in \bar{\Omega}, \\
\mathcal{V}_{M}^{n} \geqslant V_{\varepsilon}^{n}(x) \geqslant \mathcal{V}_{m}^{n} \geqslant \mathcal{V}_{m}^{0} \geqslant 0 \quad & \forall x \in \bar{\Omega}
\end{array}
$$

and

$$
\left(\theta U_{\varepsilon}^{n}+V_{\varepsilon}^{n}, 1\right)^{h}=\left(\theta U_{\varepsilon}^{0}+V_{\varepsilon}^{0}, 1\right)^{h} .
$$

Proof. For any $W \equiv \sum_{j \in J} W_{j} \chi_{j} \in S^{h}$ we define $\underline{W}:=\left(W_{1}, \ldots, W_{J}\right)^{T} \in \mathbf{R}^{\mathcal{J}}$. We then introduce $F_{U}^{n}: \mathbf{R}^{\mathcal{J}} \times \mathbf{R}^{\mathcal{J}} \rightarrow \mathbf{R}^{\mathcal{J}}$ defined by

$$
\left[F_{U}^{n}(\underline{W}, \underline{Z})\right]_{j}:=\left(W, \chi_{j}\right)^{h}+\tau_{n} c\left(\nabla W, \nabla \chi_{j}\right)+\tau_{n}\left(g(W, Z), \chi_{j}\right)^{h} \quad \forall j \in J .
$$

On noting (2.1), (1.3) and (2.10), it follows for every fixed $\underline{Z} \in \mathbf{R}^{\mathcal{J}}$ that $F_{U}^{n}(\underline{W}, \underline{Z})$ can be written as $\mathcal{A} \underline{W}+\varphi(\underline{W})$ with $\mathcal{A} \in \mathbf{R}^{\mathcal{J} \times \mathcal{J}}$ being an $\mathcal{M}$-matrix and $\varphi: \mathbf{R}^{\mathcal{J}} \rightarrow \mathbf{R}^{\overline{\mathcal{J}}}$ being a continuous isotone diagonal mapping. Hence for every fixed $\underline{Z} \in \mathbf{R}^{\mathcal{J}}$, we have that

$$
F_{U}^{n}(\cdot, \underline{Z}): \mathbf{R}^{\mathcal{J}} \rightarrow \mathbf{R}^{\mathcal{J}} \quad \text { is an inverse isotone homeomorphism, }
$$

see e.g. [10: § 13.5.6].

Given $U_{\varepsilon}^{n-1}, V_{\varepsilon}^{n-1} \in K^{h}$, and hence $\underline{U}_{\varepsilon}^{n-1}, \underline{V}_{\varepsilon}^{n-1} \in \mathbf{R}_{\geqslant 0}^{\mathcal{J}}$, (2.11a) can be rewritten as: find $\underline{U}_{\varepsilon}^{n} \in \mathbf{R}^{\mathcal{J}}$ such that

$$
F_{U}^{n}\left(\underline{U}_{\varepsilon}^{n}, \underline{V}_{\varepsilon}^{n-1}\right)=\underline{X}_{U}^{n} \in \mathbf{R}_{\geqslant 0}^{\mathcal{J}},
$$

where $\left[\underline{X}_{U}^{n}\right]_{j}:=\left(U_{\varepsilon}^{n-1}, \chi_{j}\right)^{h}$ for all $j \in J$. On noting (2.43), (2.42) and that $F_{U}^{n}\left(\underline{0}, \underline{V}_{\varepsilon}^{n-1}\right)=\underline{0}$, we have existence and uniqueness of $U_{\varepsilon}^{n} \in K^{h}$ solving (2.11a).

Given $U_{\varepsilon}^{n}, V_{\varepsilon}^{n-1} \in K^{h}$, to prove the existence of $V_{\varepsilon}^{n}$ we will make use of the Brouwer fixedpoint theorem, see e.g. [11: Theorem 9.36]. Let $\widetilde{F}_{V}^{n}: \mathbf{R}^{\mathcal{J}} \rightarrow \mathbf{R}^{\mathcal{J}}$ be defined by

$$
\left[\widetilde{F}_{V}^{n}(\underline{Z})\right]_{j}:=\left(Z, \chi_{j}\right)^{h}+\tau_{n} \sigma\left(U_{\varepsilon}^{n} \Lambda_{\varepsilon}(Z) \nabla Z, \nabla \chi_{j}\right) \quad \forall j \in J .
$$

(2.11b) can then be rewritten as: find $\underline{V}_{\varepsilon}^{n} \in \mathbf{R}^{\mathcal{J}}$ such that

$$
\widetilde{F}_{V}^{n}\left(\underline{V}_{\varepsilon}^{n}\right)=\underline{X}_{V}^{n} \in \mathbf{R}_{\geqslant 0}^{\mathcal{J}},
$$

where $\left[\underline{X}_{V}^{n}\right]_{j}:=\left(V_{\varepsilon}^{n-1}+\tau_{n} \theta g\left(U_{\varepsilon}^{n}, V_{\varepsilon}^{n-1}\right), \chi_{j}\right)^{h}$ for all $j \in J$. It follows from (2.21) that $\widetilde{F}_{V}^{n}$ is continuous and hence it is sufficient to show that $\widetilde{F}_{V}^{n}$ is coercive. On noting (2.20) and that $U_{\varepsilon}^{n} \in K^{h}$, we have that

$$
\sum_{j \in J}\left[\widetilde{F}_{V}^{n}(\underline{Z})\right]_{j} Z_{j} \equiv|Z|_{h}^{2}+\tau_{n} \sigma\left(U_{\varepsilon}^{n} \Lambda_{\varepsilon}(Z) \nabla Z, \nabla Z\right) \geqslant|Z|_{h}^{2} \quad \forall Z \in S^{h} .
$$


Hence the coerciveness of $\widetilde{F}_{V}^{n}$ follows from (2.46), (2.16) and (2.2). Therefore, on noting the aforementioned theorem and (2.45), we have existence of $V_{\varepsilon}^{n} \in S^{h}$ solving (2.11b). The fact that $V_{\varepsilon}^{n} \in K^{h}$ will be demonstrated below.

For the remainder of the proof we proceed by induction. We assume that the bounds (2.39a,b) hold for $n$ replaced by $n-1$. On setting $1:=(1, \ldots, 1)^{T} \in \mathbf{R}^{\mathcal{J}}$, it follows from (2.41), (2.32a), (2.33a), (2.43), (1.3) and (2.39a,b) with $n$ replaced by $n-1$ that for all $j \in J$

$$
\begin{aligned}
& {\left[F_{U}^{n}\left(\mathcal{U}_{M}^{n} \underline{1}, \mathcal{V}_{m}^{n-1} \underline{1}\right)-F_{U}^{n}\left(\underline{U}_{\varepsilon}^{n}, \mathcal{V}_{m}^{n-1} \underline{1}\right)\right]_{j}=} \\
& \quad\left(\mathcal{U}_{M}^{n-1}-U_{\varepsilon}^{n-1}, \chi_{j}\right)^{h}+\tau_{n}\left(g\left(U_{\varepsilon}^{n}, V_{\varepsilon}^{n-1}\right)-g\left(U_{\varepsilon}^{n}, \mathcal{V}_{m}^{n-1}\right), \chi_{j}\right)^{h} \geqslant 0, \\
& {\left[F_{U}^{n}\left(\underline{U}_{\varepsilon}^{n}, \mathcal{V}_{M}^{n-1} \underline{1}\right)-F_{U}^{n}\left(\mathcal{U}_{m}^{n} \underline{1}, \mathcal{V}_{M}^{n-1} \underline{1}\right)\right]_{j}=} \\
& \left(U_{\varepsilon}^{n-1}-\mathcal{U}_{m}^{n-1}, \chi_{j}\right)^{h}+\tau_{n}\left(g\left(U_{\varepsilon}^{n}, \mathcal{V}_{M}^{n-1}\right)-g\left(U_{\varepsilon}^{n}, V_{\varepsilon}^{n-1}\right), \chi_{j}\right)^{h} \geqslant 0 .
\end{aligned}
$$

Noting (2.47a,b) and (2.42) we obtain that the desired bounds (2.39a) hold for $n$.

Let $F_{V}^{n}: \mathbf{R}^{\mathcal{J}} \rightarrow \mathbf{R}^{\mathcal{J}}$ be defined by

$$
\left[F_{V}^{n}(\underline{Z})\right]_{j}:=\left(Z, \chi_{j}\right)^{h}+\tau_{n} \sigma\left(U_{\varepsilon}^{n} \Lambda_{\varepsilon}\left(V_{\varepsilon}^{n}\right) \nabla Z, \nabla \chi_{j}\right) \quad \forall j \in J .
$$

Hence on noting (2.48) and (2.45), the solution $V_{\varepsilon}^{n} \in S^{h}$ of (2.11b) is such that

$$
F_{V}^{n}\left(\underline{V}_{\varepsilon}^{n}\right)=\underline{X}_{V}^{n} \in \mathbf{R}_{\geqslant 0}^{\mathcal{J}}
$$

Adopting the notation of (2.8), we have for all $\kappa \in \mathcal{T}^{h}$, on noting that $R_{\kappa}^{T} \equiv R_{\kappa}^{-1}$, that

$$
\int_{\kappa} U_{\varepsilon}^{n}\left(\nabla \chi_{k}\right)^{T} \Lambda_{\varepsilon}\left(V_{\varepsilon}^{n}\right) \nabla \chi_{j} \mathrm{~d} x \equiv \sum_{i=1}^{d} \int_{\widehat{\kappa}} \widehat{U}_{\varepsilon}^{n}\left[\widehat{\Lambda}_{\varepsilon}\left(\widehat{U}_{\varepsilon}^{n}\right)\right]_{i i} \frac{\partial \widehat{\chi}_{j}}{\partial \widehat{x}_{i}} \frac{\partial \widehat{\chi}_{k}}{\partial \widehat{x}_{i}} \mathrm{~d} \widehat{x} \quad \forall j, k \in J .
$$

It follows from (2.50), $U_{\varepsilon}^{n} \in K^{h}$, (2.9) and (2.5) that $F_{V}^{n}(\underline{Z})$ can be written as $\mathcal{B Z}$ with $\mathcal{B} \in \mathbf{R}^{\mathcal{J} \times \mathcal{J}}$ being an $\mathcal{M}$-matrix. Hence $F_{V}^{n}(\cdot): \mathbf{R}^{\mathcal{J}} \rightarrow \mathbf{R}^{\mathcal{J}}$ is an inverse isotone homeomorphism, and we deduce immediately from (2.49) and $F_{V}^{n}(0)=\underline{0}$ that $V_{\varepsilon}^{n} \in K^{h}$.

It follows from (2.48), (2.32b), (2.33b), (2.49), (1.3), (2.39a) and the bounds (2.39b) with $n$ replaced by $n-1$ that for all $j \in J$

$$
\begin{aligned}
& {\left[F_{V}^{n}\left(\mathcal{V}_{M}^{n} \underline{1}\right)-F_{V}^{n}\left(\underline{V}_{\varepsilon}^{n}\right)\right]_{j}=} \\
& \left(\mathcal{V}_{M}^{n-1}-V_{\varepsilon}^{n-1}, \chi_{j}\right)^{h}+\tau_{n} \theta\left(g\left(\mathcal{U}_{M}^{n}, \mathcal{V}_{M}^{n-1}\right)-g\left(U_{\varepsilon}^{n}, V_{\varepsilon}^{n-1}\right), \chi_{j}\right)^{h} \geqslant 0 \\
& {\left[F_{V}^{n}\left(\underline{V}_{\varepsilon}^{n}\right)-F_{V}^{n}\left(\mathcal{V}_{m}^{n} \underline{1}\right)\right]_{j}=} \\
& \quad\left(V_{\varepsilon}^{n-1}-\mathcal{V}_{m}^{n-1}, \chi_{j}\right)^{h}+\tau_{n} \theta\left(g\left(\mathcal{U}_{\varepsilon}^{n}, V_{\varepsilon}^{n-1}\right)-g\left(\mathcal{U}_{m}^{n}, \mathcal{V}_{m}^{n-1}\right), \chi_{j}\right)^{h} \geqslant 0 .
\end{aligned}
$$

On noting that $F_{V}^{n}$ is inverse isotone and (2.51a,b), we obtain that the desired bounds (2.39b) hold for $n$. As the bounds (2.39a,b) hold for $n=0$, see (2.34), it follows by induction that they hold for all $n \geqslant 0$

Finally, we note on choosing $\chi \equiv 1$ in $(2.11 \mathrm{a}, \mathrm{b})$ that $\left(\theta U_{\varepsilon}^{n}+V_{\varepsilon}^{n}, 1\right)^{h}=\left(\theta U_{\varepsilon}^{n-1}+V_{\varepsilon}^{n-1}, 1\right)^{h}$ and thus the desired result (2.40) holds. 
Lemma 2.4 Let the assumptions (A) hold, $g(\cdot, \cdot)$ satisfy (1.4), and $\mathcal{U}_{M}^{0}, \mathcal{U}_{m}^{0}, \mathcal{V}_{M}^{0}, \mathcal{V}_{m}^{0}$ satisfy (2.34). Then for all $\varepsilon \in(0,1), h>0$ and for all time partitions $\left\{\tau_{n}\right\}_{n=1}^{N}$, a solution $\left\{U_{\varepsilon}^{n}, V_{\varepsilon}^{n}\right\}_{n=1}^{N}$ of $\left(\mathrm{P}_{\varepsilon}^{h, \tau}\right)$ is uniformly bounded. In particular, it holds for all $n \geqslant 0$ that

$$
\mathcal{U}_{M}^{0} \geqslant U_{\varepsilon}^{n}(x) \geqslant 0 \quad \text { and } \quad \mathcal{V}_{M} \geqslant V_{\varepsilon}^{n}(x) \geqslant \mathcal{V}_{m}^{0} \quad \forall x \in \bar{\Omega},
$$

where

$$
\mathcal{V}_{M}:=\exp \left(\theta f\left(\mathcal{U}_{M}^{0}\right) T\right) \mathcal{V}_{M}^{0} .
$$

Proof. From (2.32b), (1.4) and (2.35a,b), it follows for all $n \geqslant 1$ that

$$
\begin{aligned}
\mathcal{V}_{M}^{n} & =\mathcal{V}_{M}^{n-1}+\tau_{n} \theta g\left(\mathcal{U}_{M}^{n}, \mathcal{V}_{M}^{n-1}\right) \leqslant \mathcal{V}_{M}^{n-1}+\tau_{n} \theta g\left(\mathcal{U}_{M}^{0}, \mathcal{V}_{M}^{n-1}\right) \\
& =\mathcal{V}_{M}^{n-1}+\tau_{n} \theta f\left(\mathcal{U}_{M}^{0}\right) \mathcal{V}_{M}^{n-1} \leqslant \exp \left(\tau_{n} \theta f\left(\mathcal{U}_{M}^{0}\right)\right) \mathcal{V}_{M}^{n-1} \leqslant \exp \left(\theta f\left(\mathcal{U}_{M}^{0}\right) t_{n}\right) \mathcal{V}_{M}^{0} .
\end{aligned}
$$

The desired result (2.52a,b) then follows from (2.39a,b) and (2.53).

THEOREM 2.2 Let the assumptions of Lemma 2.4 hold. Then for all $\varepsilon \in(0,1), h>0$ and for all time partitions $\left\{\tau_{n}\right\}_{n=1}^{N}$, a solution $\left\{U_{\varepsilon}^{n}, V_{\varepsilon}^{n}\right\}_{n=1}^{N}$ of $\left(\mathrm{P}_{\varepsilon}^{h, \tau}\right)$ satisfies

$$
\begin{aligned}
\sum_{n=1}^{N} \tau_{n} & \left|\frac{U_{\varepsilon}^{n}-U_{\varepsilon}^{n-1}}{\tau_{n}}\right|_{h}^{2}+c \max _{1 \leqslant n \leqslant N}\left|U_{\varepsilon}^{n}\right|_{1}^{2}+c \sum_{n=1}^{N}\left|U_{\varepsilon}^{n}-U_{\varepsilon}^{n-1}\right|_{1}^{2}+\sum_{n=1}^{N}\left|V_{\varepsilon}^{n}-V_{\varepsilon}^{n-1}\right|_{h}^{2} \\
& +\sigma \sum_{n=1}^{N} \tau_{n}\left(U_{\varepsilon}^{n} \Lambda_{\varepsilon}\left(V_{\varepsilon}^{n}\right) \nabla V_{\varepsilon}^{n}, \nabla V_{\varepsilon}^{n}\right)+\sigma \sum_{n=1}^{N} \tau_{n}\left(U_{\varepsilon}^{n} \nabla V_{\varepsilon}^{n}, \nabla V_{\varepsilon}^{n}\right)+\max _{1 \leqslant n \leqslant N}\left(\mathcal{L}_{\varepsilon}\left(V_{\varepsilon}^{n}\right), 1\right)^{h} \\
& +\sum_{n=1}^{N} \tau_{n}\left\|\frac{V_{\varepsilon}^{n}-V_{\varepsilon}^{n-1}}{\tau_{n}}\right\|_{-1}^{2} \leqslant C\left|U_{\varepsilon}^{0}\right|_{1}^{2}+C\left(\mathcal{U}_{M}^{0}, \mathcal{V}_{M}^{0}, T\right)
\end{aligned}
$$

Proof. Choosing $\chi \equiv\left(U_{\varepsilon}^{n}-U_{\varepsilon}^{n-1}\right)$ in (2.11a) yields that

$$
\tau_{n}\left|\frac{U_{\varepsilon}^{n}-U_{\varepsilon}^{n-1}}{\tau_{n}}\right|_{h}^{2}+c\left(\nabla U_{\varepsilon}^{n}, \nabla\left(U_{\varepsilon}^{n}-U_{\varepsilon}^{n-1}\right)\right)+\left(g\left(U_{\varepsilon}^{n}, V_{\varepsilon}^{n-1}\right), U_{\varepsilon}^{n}-U_{\varepsilon}^{n-1}\right)^{h}=0 .
$$

On noting (1.15), (1.16), (1.4) and (2.52a,b) it follows that

$$
\begin{aligned}
\tau_{n}\left|\frac{U_{\varepsilon}^{n}-U_{\varepsilon}^{n-1}}{\tau_{n}}\right|_{h}^{2}+c\left|U_{\varepsilon}^{n}\right|_{1}^{2}+c\left|U_{\varepsilon}^{n}-U_{\varepsilon}^{n-1}\right|_{1}^{2} & \leqslant c\left|U_{\varepsilon}^{n-1}\right|_{1}^{2}+\tau_{n}\left|g\left(U_{\varepsilon}^{n}, V_{\varepsilon}^{n-1}\right)\right|_{h}^{2} \\
& \leqslant c\left|U_{\varepsilon}^{n-1}\right|_{1}^{2}+\tau_{n} C\left(\mathcal{U}_{M}^{0}, \mathcal{V}_{M}^{0}, T\right) .
\end{aligned}
$$

Summing (2.56) from $n=1, \ldots, k$, for $k=1, \ldots, N$, yields the first three bounds in (2.54).

Choosing $\chi \equiv V_{\varepsilon}^{n}$ in (2.11b) and applying (1.16), (1.4) and (2.52a,b) we have that

$$
\begin{aligned}
\frac{1}{2}\left|V_{\varepsilon}^{n}\right|_{h}^{2}+\frac{1}{2}\left|V_{\varepsilon}^{n}-V_{\varepsilon}^{n-1}\right|_{h}^{2}+\tau_{n} \sigma\left(U_{\varepsilon}^{n} \Lambda_{\varepsilon}\left(V_{\varepsilon}^{n}\right)\right. & \left.\nabla V_{\varepsilon}^{n}, \nabla V_{\varepsilon}^{n}\right) \\
& =\frac{1}{2}\left|V_{\varepsilon}^{n-1}\right|_{h}^{2}+\tau_{n} \theta\left(g\left(U_{\varepsilon}^{n}, V_{\varepsilon}^{n-1}\right), V_{\varepsilon}^{n}\right)^{h} \\
& \leqslant \frac{1}{2}\left|V_{\varepsilon}^{n-1}\right|_{h}^{2}+\tau_{n} C\left(\mathcal{U}_{M}^{0}, \mathcal{V}_{M}^{0}, T\right) .
\end{aligned}
$$


Summing (2.57) from $n=1, \ldots, N$ yields the fourth and fifth bound in (2.54).

Choosing $\chi \equiv \pi^{h}\left[\mathcal{L}_{\varepsilon}^{\prime}\left(V_{\varepsilon}^{n}\right)\right]$ in (2.11b) and noting (2.6a,b) and (2.5) yields that

$$
\left(\mathcal{L}_{\varepsilon}\left(V_{\varepsilon}^{n}\right)-\mathcal{L}_{\varepsilon}\left(V_{\varepsilon}^{n-1}\right), 1\right)^{h}+\tau_{n} \sigma\left(U_{\varepsilon}^{n} \nabla V_{\varepsilon}^{n}, \nabla V_{\varepsilon}^{n}\right) \leqslant \tau_{n} \theta\left(g\left(U_{\varepsilon}^{n}, V_{\varepsilon}^{n-1}\right), \mathcal{L}_{\varepsilon}^{\prime}\left(V_{\varepsilon}^{n}\right)\right)^{h} .
$$

Summing (2.58) from $n=1, \ldots, k$, for $k=1 \rightarrow N$, and noting (1.4), (2.52a,b) and that $\mathcal{L}_{\varepsilon}^{\prime}\left(V_{\varepsilon}^{n}\right) \leqslant$ $\left[\ln \mathcal{V}_{M}\right]_{+}$yields the sixth and seventh bound in (2.54).

Finally from (2.3), (2.11b), (2.52a,b), (2.20), (2.16), (2.19), (1.4) and on recalling from (2.6a) that $\Lambda_{\varepsilon}$ is positive semidefinite we obtain that

$$
\begin{aligned}
\left(\frac{V_{\varepsilon}^{n}-V_{\varepsilon}^{n-1}}{\tau_{n}}, \eta\right) & =\left(\frac{V_{\varepsilon}^{n}-V_{\varepsilon}^{n-1}}{\tau_{n}}, Q^{h} \eta\right)^{h} \\
& =-\sigma\left(U_{\varepsilon}^{n} \Lambda_{\varepsilon}\left(V_{\varepsilon}^{n}\right) \nabla V_{\varepsilon}^{n}, \nabla\left(Q^{h} \eta\right)\right)+\theta\left(g\left(U_{\varepsilon}^{n}, V_{\varepsilon}^{n-1}\right), Q^{h} \eta\right)^{h} \\
& \leqslant C\left(\mathcal{U}_{M}^{0}\right)\left[\left|\left[U_{\varepsilon}^{n} \Lambda_{\varepsilon}\left(V_{\varepsilon}^{n}\right)\right]^{\frac{1}{2}} \nabla V_{\varepsilon}^{n}\right|_{0}+\left|g\left(U_{\varepsilon}^{n}, V_{\varepsilon}^{n-1}\right)\right|_{h}\right]\left\|Q^{h} \eta\right\|_{1} \\
& \leqslant C\left(\mathcal{U}_{M}^{0}, \mathcal{V}_{M}^{0}, T\right)\left[\left|\left[U_{\varepsilon}^{n} \Lambda_{\varepsilon}\left(V_{\varepsilon}^{n}\right)\right]^{\frac{1}{2}} \nabla V_{\varepsilon}^{n}\right|_{0}+1\right]\|\eta\|_{1} \quad \forall \eta \in H^{1}(\Omega)
\end{aligned}
$$

and hence that

$$
\left\|\frac{V_{\varepsilon}^{n}-V_{\varepsilon}^{n-1}}{\tau_{n}}\right\|_{-1}^{2} \leqslant C\left(\mathcal{U}_{M}^{0}, \mathcal{V}_{M}^{0}, T\right)\left[\left|\left[U_{\varepsilon}^{n} \Lambda_{\varepsilon}\left(V_{\varepsilon}^{n}\right)\right]^{\frac{1}{2}} \nabla V_{\varepsilon}^{n}\right|_{0}^{2}+1\right] .
$$

Multiplying (2.59b) by $\tau_{n}$, summing from $n=1, \ldots, N$, and noting the fifth bound in (2.54) yields the final bound in (2.54).

LEMMA 2.5 Let $u^{0}, v^{0} \in K \cap L^{\infty}(\Omega)$ and the assumptions (A) hold. On choosing either $U_{\varepsilon}^{0} \equiv$ $Q^{h} u^{0}, V_{\varepsilon}^{0} \equiv Q^{h} v^{0}$ or in the case $d=1 U_{\varepsilon}^{0} \equiv \pi^{h} u^{0}, V_{\varepsilon}^{0} \equiv \pi^{h} v^{0}$; it follows that $U_{\varepsilon}^{0}, V_{\varepsilon}^{0} \in K^{h}$ and $\mathcal{U}_{M}^{0} \equiv \sup _{x \in \Omega} u^{0}(x), \mathcal{V}_{M}^{0} \equiv \sup _{x \in \Omega} v^{0}(x)$ satisfying (2.34) are such that for all $h>0$ and $\varepsilon \in(0,1)$

$$
\left|U_{\varepsilon}^{0}\right|_{1}^{2}+C\left(\mathcal{U}_{M}^{0}, \mathcal{V}_{M}^{0}, T\right) \leqslant C(T)
$$

Proof. The desired result follows immediately from (2.19), (2.14), (2.34) and (2.18).

\section{Convergence}

Let

$$
U_{\varepsilon}(t):=\frac{t-t_{n-1}}{\tau_{n}} U_{\varepsilon}^{n}+\frac{t_{n}-t}{\tau_{n}} U_{\varepsilon}^{n-1} \quad t \in\left[t_{n-1}, t_{n}\right] \quad n \geqslant 1
$$

and

$$
U_{\varepsilon}^{+}(t):=U_{\varepsilon}^{n}, \quad U_{\varepsilon}^{-}(t):=U_{\varepsilon}^{n-1} \quad t \in\left(t_{n-1}, t_{n}\right] \quad n \geqslant 1 .
$$


Using the above notation, and introducing analogous notation for $V_{\varepsilon}$, and $(2.39 \mathrm{a}, \mathrm{b})$; then $(2.11 \mathrm{a}, \mathrm{b})$ can be restated as: find $\left\{U_{\varepsilon}, V_{\varepsilon}\right\} \in C\left([0, T] ; K^{h}\right) \times C\left([0, T] ; K^{h}\right)$ such that

$$
\begin{gathered}
\int_{0}^{T}\left[\left(\frac{\partial U_{\varepsilon}}{\partial t}, \chi\right)^{h}+c\left(\nabla U_{\varepsilon}^{+}, \nabla \chi\right)\right] \mathrm{d} t=-\int_{0}^{T}\left(g\left(U_{\varepsilon}^{+}, V_{\varepsilon}^{-}\right), \chi\right)^{h} \mathrm{~d} t \\
\forall \chi \in L^{2}\left(0, T ; S^{h}\right), \\
\int_{0}^{T}\left[\left(\frac{\partial V_{\varepsilon}}{\partial t}, \chi\right)^{h}+\sigma\left(U_{\varepsilon}^{+} \Lambda_{\varepsilon}\left(V_{\varepsilon}^{+}\right) \nabla V_{\varepsilon}^{+}, \nabla \chi\right)\right] \mathrm{d} t=\theta \int_{0}^{T}\left(g\left(U_{\varepsilon}^{+}, V_{\varepsilon}^{-}\right), \chi\right)^{h} \mathrm{~d} t \\
\forall \chi \in L^{2}\left(0, T ; S^{h}\right) .
\end{gathered}
$$

Lemma 3.1 Let $u^{0}, v^{0} \in K \cap L^{\infty}(\Omega), g(\cdot, \cdot)$ satisfy (1.4) and $\left\{\mathcal{T}^{h}, U_{\varepsilon}^{0}, V_{\varepsilon}^{0}, \tau, \varepsilon\right\}_{h>0}$ be such that

(i) either $U_{\varepsilon}^{0} \equiv Q^{h} u^{0}, V_{\varepsilon}^{0} \equiv Q^{h} v^{0}$ or in the case $d=1 U_{\varepsilon}^{0} \equiv \pi^{h} u^{0}, V_{\varepsilon}^{0} \equiv \pi^{h} v^{0}$;

(ii) $\Omega$ and $\left\{\mathcal{T}^{h}\right\}_{h>0}$ fulfil assumption (A), $\varepsilon \in(0,1)$ and $\tau_{n} \leqslant C \tau_{n-1}, n=2, \ldots, N$;

(iii) $\varepsilon, \tau \rightarrow 0$ as $h \rightarrow 0$.

Then there exists a subsequence of $\left\{U_{\varepsilon}, V_{\varepsilon}\right\}_{h}$ and functions

$$
\begin{aligned}
& u \in L^{\infty}\left(\Omega_{T}\right) \cap L^{\infty}(0, T ; K) \cap H^{1}\left(0, T ; L^{2}(\Omega)\right) \\
\text { and } \quad & v \in\left\{\eta \in L^{\infty}\left(\Omega_{T}\right): \eta \geqslant 0 \text { a.e. in } \Omega_{T}\right\} \cap H^{1}\left(0, T ;\left(H^{1}(\Omega)\right)^{\prime}\right) \\
\text { such that } \quad & u v \in L^{2}\left(0, T ; H^{1}(\Omega)\right),
\end{aligned}
$$

and as $h \rightarrow 0$

$$
\begin{aligned}
U_{\varepsilon}, U_{\varepsilon}^{ \pm} \rightarrow u & \text { weak-* in } L^{\infty}\left(\Omega_{T}\right), \\
\frac{\partial U_{\varepsilon}}{\partial t} \rightarrow \frac{\partial u}{\partial t} & \text { weakly in } L^{2}\left(\Omega_{T}\right), \\
U_{\varepsilon}, U_{\varepsilon}^{ \pm} \rightarrow u & \text { weak-* in } L^{\infty}\left(0, T ; H^{1}(\Omega)\right), \\
U_{\varepsilon}, U_{\varepsilon}^{ \pm} \rightarrow u & \text { strongly in } L^{p}\left(\Omega_{T}\right) \text { and a.e. in } \Omega_{T}, \\
& \\
V_{\varepsilon}, V_{\varepsilon}^{ \pm} \rightarrow v & \text { weak-* in } L^{\infty}\left(\Omega_{T}\right), \\
\frac{\partial V_{\varepsilon}}{\partial t} \rightarrow \frac{\partial v}{\partial t} & \text { weakly in } L^{2}\left(0, T ;\left(H^{1}(\Omega)\right)^{\prime}\right), \\
f\left(U_{\varepsilon}^{+}\right) V_{\varepsilon}^{-} \rightarrow f(u) v & \text { weak-* in } L^{\infty}\left(\Omega_{T}\right), \\
U_{\varepsilon} V_{\varepsilon}, U_{\varepsilon}^{ \pm} V_{\varepsilon}^{ \pm} \rightarrow u v & \text { strongly in } L^{p}\left(\Omega_{T}\right) \text { and a.e. in } \Omega_{T}, \\
U_{\varepsilon}^{ \pm} V_{\varepsilon}^{ \pm} \rightarrow u v & \text { weakly in } L^{2}\left(0, T ; H^{1}(\Omega)\right),
\end{aligned}
$$

for any $p \in[1, \infty)$. In addition it holds that $f[\theta u(\cdot, t)+v(\cdot, t)]=f\left[\theta u^{0}+v^{0}\right]$ for a.e. $t \in[0, T]$. 
Proof. It follows from the definitions (3.1a,b) and the bounds (2.54), as $c, \sigma>0$, together with (2.16), (2.52a,b), assumptions (i) and (ii), and (2.60) that

$$
\begin{aligned}
\left\|U_{\varepsilon}^{( \pm)}\right\|_{L^{\infty}\left(\Omega_{T}\right)}^{2}+\left\|U_{\varepsilon}^{( \pm)}\right\|_{L^{\infty}\left(0, T ; H^{1}(\Omega)\right)}^{2}+\left\|\frac{\partial U_{\varepsilon}}{\partial t}\right\|_{L^{2}\left(\Omega_{T}\right)}+\tau^{-1}\left\|U_{\varepsilon}^{+}-U_{\varepsilon}^{-}\right\|_{L^{2}\left(0, T ; H^{1}(\Omega)\right)}^{2} \\
\quad+\left\|V_{\varepsilon}^{( \pm)}\right\|_{L^{\infty}\left(\Omega_{T}\right)}^{2}+\tau^{-1}\left\|V_{\varepsilon}^{+}-V_{\varepsilon}^{-}\right\|_{L^{2}\left(\Omega_{T}\right)}^{2}+\left\|\left[U_{\varepsilon}^{+} \Lambda_{\varepsilon}\left(V_{\varepsilon}^{+}\right)\right]^{\frac{1}{2}} \nabla V_{\varepsilon}^{+}\right\|_{L^{2}\left(\Omega_{T}\right)}^{2} \\
\quad+\left\|\left(U_{\varepsilon}^{+}\right)^{\frac{1}{2}} \nabla V_{\varepsilon}^{+}\right\|_{L^{2}\left(\Omega_{T}\right)}^{2}+\left\|\frac{\partial V_{\varepsilon}}{\partial t}\right\|_{L^{2}\left(0, T ;\left(H^{1}(\Omega)\right)^{\prime}\right)} \leqslant C(T)
\end{aligned}
$$

where $U_{\varepsilon}^{( \pm)}$is an abbreviation for 'with' and 'without' the superscripts '+' and '-'; similarly, $V_{\varepsilon}^{( \pm)}$. Hence on noting (3.7), (3.1a,b), (2.39a,b), (2.40), assumptions (i) and (iii), (2.3) and (2.14) we can choose a subsequence $\left\{U_{\varepsilon}, V_{\varepsilon}\right\}_{h}$ such that the convergence results $(3.3 \mathrm{a}, \mathrm{b}),(3.4 \mathrm{a}-\mathrm{c})$ and $(3.5 \mathrm{a}, \mathrm{b})$ hold and the additional integral condition is satisfied. The convergence result (3.4d) for $p=2$ follows immediately on extracting a further subsequence from $(3.4 \mathrm{~b}, \mathrm{c}),(1.14 \mathrm{a})$, the fourth bound in (3.7) and the assumption (iii). The result (3.4d) for any $p \in[1, \infty)$ then follows from the a.e. convergence and (3.3a). The a.e. convergence (3.4d), together with (1.4) and (3.3a) imply for any $p \in[1, \infty)$ that

$$
f(u) \in L^{\infty}\left(\Omega_{T}\right) \quad \text { and } \quad f\left(U_{\varepsilon}^{( \pm)}\right) \rightarrow f(u) \quad \text { a.e. in } \Omega_{T} \text { and strongly in } L^{p}\left(\Omega_{T}\right) .
$$

Combining (3.8) and (3.5a) yields the desired result (3.6a).

Similarly to the proof of (3.6a), we have that

$$
U_{\varepsilon} V_{\varepsilon}, U_{\varepsilon}^{ \pm} V_{\varepsilon}^{ \pm} \rightarrow u v \quad \text { weak-* in } L^{\infty}\left(\Omega_{T}\right)
$$

To prove $(3.6 \mathrm{~b})$, we introduce

$$
W_{\varepsilon}^{n}:=U_{\varepsilon}^{n} V_{\varepsilon}^{n} \notin S^{h} \quad n=0, \ldots, N
$$

and define $W_{\varepsilon}^{( \pm)}$similarly to $U_{\varepsilon}^{( \pm)}$as in (3.1a,b). It then follows from (3.10) and (3.7) for any $\eta \in L^{2}\left(0, T ; W^{1, q}(\Omega)\right)$, with $q=2$ if $d=1$ and $q>2$ if $d=2$, that

$$
\begin{aligned}
& \left|\int_{0}^{T}\left(\frac{\partial W_{\varepsilon}}{\partial t}, \eta\right) \mathrm{d} t\right|=\left|\int_{0}^{T}\left(V_{\varepsilon}^{+} \frac{\partial U_{\varepsilon}}{\partial t}+U_{\varepsilon}^{-} \frac{\partial V_{\varepsilon}}{\partial t}, \eta\right) \mathrm{d} t\right| \\
& \quad \leqslant C\left[\left\|\frac{\partial U_{\varepsilon}}{\partial t}\right\|_{L^{2}\left(\Omega_{T}\right)}\|\eta\|_{L^{2}\left(\Omega_{T}\right)}+\left\|\frac{\partial V_{\varepsilon}}{\partial t}\right\|_{L^{2}\left(0, T ;\left(H^{1}(\Omega)\right)^{\prime}\right)}\left\|U_{\varepsilon}^{-} \eta\right\|_{L^{2}\left(0, T ; H^{1}(\Omega)\right)}\right] \\
& \quad \leqslant C\|\eta\|_{L^{2}\left(0, T ; W^{1, q}(\Omega)\right)}
\end{aligned}
$$

where we have noted from (1.13) that

$$
\begin{aligned}
\left\|\nabla\left(U_{\varepsilon}^{-} \eta\right)\right\|_{L^{2}\left(\Omega_{T}\right)} & \leqslant\left\|U_{\varepsilon}^{-}\right\|_{L^{\infty}\left(0, T ; H^{1}(\Omega)\right)}\|\eta\|_{L^{2}\left(0, T ; L^{\infty}(\Omega)\right)}+\left\|U_{\varepsilon}^{-}\right\|_{L^{\infty}\left(\Omega_{T}\right)}\|\eta\|_{L^{2}\left(0, T ; H^{1}(\Omega)\right)} \\
& \leqslant C\|\eta\|_{L^{2}\left(0, T ; W^{1, q}(\Omega)\right)} .
\end{aligned}
$$


We have from (3.10), (3.1a,b) and (3.7) that

$$
\begin{aligned}
\left\|\nabla W_{\varepsilon}\right\|_{L^{2}\left(\Omega_{T}\right)}^{2} & \leqslant\left\|\nabla W_{\varepsilon}^{+}\right\|_{L^{2}\left(\Omega_{T}\right)}^{2}+\left\|\nabla W_{\varepsilon}^{-}\right\|_{L^{2}\left(\Omega_{T}\right)}^{2} \\
& \leqslant 2\left[\left\|V_{\varepsilon}^{+} \nabla U_{\varepsilon}^{+}\right\|_{L^{2}\left(\Omega_{T}\right)}^{2}+\left\|V_{\varepsilon}^{-} \nabla U_{\varepsilon}^{-}\right\|_{L^{2}\left(\Omega_{T}\right)}^{2}\right. \\
& \left.\quad+\left\|U_{\varepsilon}^{+} \nabla V_{\varepsilon}^{+}\right\|_{L^{2}\left(\Omega_{T}\right)}^{2}+\left\|U_{\varepsilon}^{-} \nabla V_{\varepsilon}^{-}\right\|_{L^{2}\left(\Omega_{T}\right)}^{2}\right] \\
& \leqslant C\left[1+\left\|\left(U_{\varepsilon}^{-}\right)^{\frac{1}{2}} \nabla V_{\varepsilon}^{-}\right\|_{L^{2}\left(\Omega_{T}\right)}^{2}\right] \leqslant C
\end{aligned}
$$

where we have noted from (2.54), our assumption (i), (2.60), the time step control (ii), (2.19) and (2.14) that

$$
\begin{aligned}
\left\|\left(U_{\varepsilon}^{-}\right)^{\frac{1}{2}} \nabla V_{\varepsilon}^{-}\right\|_{L^{2}\left(\Omega_{T}\right)}^{2} & \equiv \sum_{n=1}^{N} \tau_{n}\left|\left(U_{\varepsilon}^{n-1}\right)^{\frac{1}{2}} \nabla V_{\varepsilon}^{n-1}\right|_{0}^{2} \equiv \sum_{n=0}^{N-1} \tau_{n+1}\left|\left(U_{\varepsilon}^{n}\right)^{\frac{1}{2}} \nabla V_{\varepsilon}^{n}\right|_{0}^{2} \\
& \leqslant C\left|\nabla V_{\varepsilon}^{0}\right|_{0}^{2}+C \sum_{n=1}^{N-1} \tau_{n}\left|\left(U_{\varepsilon}^{n}\right)^{\frac{1}{2}} \nabla V_{\varepsilon}^{n}\right|_{0}^{2} \leqslant C .
\end{aligned}
$$

Combining (3.11), (3.14) and (3.7) yields that

$$
\left\|W_{\varepsilon}\right\|_{L^{2}\left(0, T ; H^{1}(\Omega)\right)}+\left\|\frac{\partial W_{\varepsilon}}{\partial t}\right\|_{L^{2}\left(0, T ;\left(W^{1, q}(\Omega)\right)^{\prime}\right)} \leqslant C .
$$

The bounds (3.15) and (1.14a) imply the existence of a subsequence $\left\{W_{\varepsilon}\right\}_{h}$ such that

$$
W_{\varepsilon} \rightarrow w \quad \text { strongly in } L^{2}\left(\Omega_{T}\right), \quad \text { weakly in } L^{2}\left(0, T ; H^{1}(\Omega)\right) .
$$

Next we note from (3.10), (3.1a,b), (3.7) and (iii) that

$$
\begin{gathered}
\left\|W_{\varepsilon}-W_{\varepsilon}^{ \pm}\right\|_{L^{2}\left(\Omega_{T}\right)}+\left\|U_{\varepsilon} V_{\varepsilon}-W_{\varepsilon}^{ \pm}\right\|_{L^{2}\left(\Omega_{T}\right)} \leqslant C\left\|U_{\varepsilon}^{+}-U_{\varepsilon}^{-}\right\|_{L^{2}\left(\Omega_{T}\right)}+C\left\|V_{\varepsilon}^{+}-V_{\varepsilon}^{-}\right\|_{L^{2}\left(\Omega_{T}\right)} \\
\rightarrow 0 \text { as } h \rightarrow 0 .
\end{gathered}
$$

Combining (3.16), (3.17), (3.9) and (3.10) yields the desired result (3.6b) for $p=2$ on extracting a further subsequence. The result $(3.6 \mathrm{~b})$ for any $p \in[1, \infty)$ then follows from the a.e. convergence and (3.3a,b). Finally, the results (3.3c) and (3.6c) follow from (3.16), (3.13), (3.10) and (3.6b).

Before proving our convergence theorem, we make a final assumption on $f(\cdot)$ and $\Omega$ :

$$
f \in C^{0,1}\left[0, \mathcal{U}_{M}^{0}\right] \quad \text { and } \quad \Omega \text { is convex. }
$$

It is easily established from (3.18) and (2.1) that

$$
\left|\pi^{h}[f(\chi)]\right|_{1} \leqslant C_{f}|\chi|_{1} \quad \forall \chi \in K^{h} \quad \text { with } \max _{x \in \bar{\Omega}} \chi(x) \leqslant \mathcal{U}_{M}^{0},
$$

where $C_{f}$ is the Lipschitz constant of $f$.

THEOREM 3.1 Let the assumptions of Lemma 3.1 and (3.18) hold. In addition let $u^{0} \in H^{2}(\Omega)$ if $d=2$. Then there exist functions $u$ and $v$ satisfying (3.3a-c) and

$$
u \in L^{\infty}\left(0, T ; H^{d}(\Omega)\right) \cap C\left(\bar{\Omega}_{T}\right), \quad \nabla v \in L_{\mathrm{loc}}^{2}(\{u>0\}),
$$


where $\{u>0\} \subset \bar{\Omega}_{T}$ is the open set defined by

$$
\{u>0\}:=\left\{(x, t) \in \bar{\Omega}_{T}: u(x, t)>0\right\} ;
$$

and there exists a subsequence of $\left\{U_{\varepsilon}, V_{\varepsilon}\right\}_{h}$ satisfying (3.4a-d), (3.5a-d) and as $h \rightarrow 0$

$$
\begin{array}{cc}
V_{\varepsilon}, V_{\varepsilon}^{ \pm} \rightarrow v & \text { strongly in } L^{p}(\{u>0\}), p \in[1, \infty), \\
& \text { and a.e. in }\{u>0\}, \\
U_{\varepsilon}^{+} \Lambda_{\varepsilon}\left(V_{\varepsilon}^{+}\right) \nabla V_{\varepsilon}^{+} \rightarrow \mathcal{H}_{\{u>0\}} u v \nabla v & \text { weakly in } L^{2}\left(\Omega_{T}\right),
\end{array}
$$

where $\mathcal{H}_{\{u>0\}}$ is the characteristic function of the set $\{u>0\}$.

Moreover $u$ and $v$ fulfil $u(\cdot, 0)=u^{0}(\cdot), v(\cdot, 0)=v^{0}(\cdot)$ and

$$
\begin{gathered}
\int_{0}^{T}\left[\left(\frac{\partial u}{\partial t}, \eta\right)+c(\nabla u, \nabla \eta)\right] \mathrm{d} t=-\int_{0}^{T}(f(u) v, \eta) \mathrm{d} t \\
\forall \eta \in L^{2}\left(0, T ; H^{1}(\Omega)\right), \\
\int_{0}^{T}\left\langle\frac{\partial v}{\partial t}, \eta\right\rangle \mathrm{d} t+\sigma \int_{\{u>0\}} u v \nabla v . \nabla \eta \mathrm{d} x \mathrm{~d} t=\theta \int_{0}^{T}(f(u) v, \eta) \mathrm{d} t \\
\forall \eta \in L^{2}\left(0, T ; H^{1}(\Omega)\right) ;
\end{gathered}
$$

where $\langle\cdot, \cdot\rangle$ denotes the duality pairing between $\left(H^{1}(\Omega)\right)^{\prime}$ and $H^{1}(\Omega)$.

Proof. For any $\eta \in L^{2}\left(0, T ; H^{2}(\Omega)\right)$, we choose $\chi \equiv \pi^{h} \eta$ in (3.2a) and now analyse the subsequent terms. On noting (2.13) and (3.7), we have that

$$
\begin{aligned}
\left|\int_{0}^{T}\left(\nabla U_{\varepsilon}^{+}, \nabla\left(I-\pi^{h}\right) \eta\right) \mathrm{d} t\right| & \leqslant C h\left\|U_{\varepsilon}^{+}\right\|_{L^{2}\left(0, T ; H^{1}(\Omega)\right)}\|\eta\|_{L^{2}\left(0, T ; H^{2}(\Omega)\right)} \\
& \leqslant C h\|\eta\|_{L^{2}\left(0, T ; H^{2}(\Omega)\right)} \quad \forall \eta \in L^{2}\left(0, T ; H^{2}(\Omega)\right) .
\end{aligned}
$$

It follows from (3.24) and (3.4c) that

$$
\int_{0}^{T}\left(\nabla U_{\varepsilon}^{+}, \nabla\left(\pi^{h} \eta\right)\right) \mathrm{d} t \rightarrow \int_{0}^{T}(\nabla u, \nabla \eta) \mathrm{d} t \quad \text { as } h \rightarrow 0, \quad \forall \eta \in L^{2}\left(0, T ; H^{2}(\Omega)\right) .
$$

We have from (2.17) and (2.13) that for any $Y \in L^{2}\left(0, T ; S^{h}\right)$ and $\eta \in L^{2}\left(0, T ; H^{2}(\Omega)\right)$

$$
\begin{aligned}
\left|\int_{0}^{T}\left[\left(Y, \pi^{h} \eta\right)^{h}-(Y, \eta)\right] \mathrm{d} t\right| & \leqslant \int_{0}^{T}\left[\left|\left(Y, \pi^{h} \eta\right)^{h}-\left(Y, \pi^{h} \eta\right)\right|+\left|\left(Y,\left(I-\pi^{h}\right) \eta\right)\right|\right] \mathrm{d} t \\
& \leqslant C h\|Y\|_{L^{2}\left(\Omega_{T}\right)}\left[\left\|\pi^{h} \eta\right\|_{L^{2}\left(0, T ; H^{1}(\Omega)\right)}+h\|\eta\|_{L^{2}\left(0, T ; H^{2}(\Omega)\right)}\right] \\
& \leqslant C h\|Y\|_{L^{2}\left(\Omega_{T}\right)}\|\eta\|_{L^{2}\left(0, T ; H^{2}(\Omega)\right)} .
\end{aligned}
$$

Combining (3.26) for $Y \equiv \frac{\partial U_{\varepsilon}}{\partial t},(3.7)$ and (3.4b) yields that

$$
\int_{0}^{T}\left(\frac{\partial U_{\varepsilon}}{\partial t}, \pi^{h} \eta\right)^{h} \mathrm{~d} t \rightarrow \int_{0}^{T}\left(\frac{\partial u}{\partial t}, \eta\right) \mathrm{d} t \quad \text { as } h \rightarrow 0, \quad \forall \eta \in L^{2}\left(0, T ; H^{2}(\Omega)\right) .
$$


It follows from (1.13) that

$$
\begin{array}{r}
\left|\int_{0}^{T}\left(\left(I-\pi^{h}\right)\left[f\left(U_{\varepsilon}^{+}\right) V_{\varepsilon}^{-}\right], \eta\right) \mathrm{d} t\right| \leqslant C\left\|\left(I-\pi^{h}\right)\left[f\left(U_{\varepsilon}^{+}\right) V_{\varepsilon}^{-}\right]\right\|_{L^{2}\left(0, T ; L^{1}(\Omega)\right)}\|\eta\|_{L^{2}\left(0, T ; H^{2}(\Omega)\right)} \\
\forall \eta \in L^{2}\left(0, T ; H^{2}(\Omega)\right) .
\end{array}
$$

Next we note from (3.7), (2.15), (2.17), (2.52a) and (3.19) that

$$
\begin{aligned}
\|\left(I-\pi^{h}\right)[ & \left.f\left(U_{\varepsilon}^{+}\right) V_{\varepsilon}^{-}\right] \|_{L^{2}\left(0, T ; L^{1}(\Omega)\right)} \\
& \leqslant\left\|V_{\varepsilon}^{-}\left(I-\pi^{h}\right)\left[f\left(U_{\varepsilon}^{+}\right)\right]\right\|_{L^{2}\left(0, T ; L^{1}(\Omega)\right)}+\left\|\left(I-\pi^{h}\right)\left[V_{\varepsilon}^{-} \pi^{h}\left(f\left(U_{\varepsilon}^{+}\right)\right)\right]\right\|_{L^{2}\left(0, T ; L^{1}(\Omega)\right)} \\
& \leqslant C h\left\|\pi^{h}\left[f\left(U_{\varepsilon}^{+}\right)\right]\right\|_{L^{2}\left(0, T ; H^{1}(\Omega)\right)} \leqslant C h\left[1+\left\|U_{\varepsilon}^{+}\right\|_{L^{2}\left(0, T ; H^{1}(\Omega)\right)}\right] \leqslant C h .
\end{aligned}
$$

Combining (3.26) for $Y \equiv \pi^{h}\left[f\left(U_{\varepsilon}^{+}\right) V_{\varepsilon}^{-}\right]$, (3.7), (3.28), (3.29) and (3.6a) yields that

$$
\int_{0}^{T}\left(f\left(U_{\varepsilon}^{+}\right) V_{\varepsilon}^{-}, \pi^{h} \eta\right)^{h} \mathrm{~d} t \rightarrow \int_{0}^{T}(f(u) v, \eta) \mathrm{d} t \quad \text { as } h \rightarrow 0, \quad \forall \eta \in L^{2}\left(0, T ; H^{2}(\Omega)\right) .
$$

It follows from (3.2a), (1.4), (3.25), (3.27), (3.30), the denseness of $L^{2}\left(0, T ; H^{2}(\Omega)\right)$ in $L^{2}\left(0, T ; H^{1}(\Omega)\right)$ and (3.7) that (3.23a) holds.

On noting (3.3a-c) we have that $u|\nabla v| \in L^{2}\left(\Omega_{T}\right)$, which together with (1.4) and (3.18) yields that

$$
\begin{aligned}
\|\nabla(f(u) v)\|_{L^{2}\left(\Omega_{T}\right)} & =\left\|f^{\prime}(u) v \nabla u+f(u) \nabla v\right\|_{L^{2}\left(\Omega_{T}\right)} \\
& \leqslant C_{f}\|v \nabla u\|_{L^{2}\left(\Omega_{T}\right)}+C_{f}\|u \nabla v\|_{L^{2}\left(\Omega_{T}\right)} \leqslant C .
\end{aligned}
$$

Combining (3.31), (1.4) and (3.3a,b) we obtain that $f(u) v \in L^{2}\left(0, T ; H^{1}(\Omega)\right)$. A simple energy estimate for (3.23a) then yields for $d=2$, on recalling our assumptions on $\Omega$ and the initial data, that $u \in L^{\infty}\left(0, T ; H^{2}(\Omega)\right)$. This can be easily seen by choosing $\eta \equiv-\mathcal{H}_{\left\{t^{\star}>t\right\}} \Delta\left(\frac{\partial u}{\partial t}\right)$ in (3.23a) for all $t^{\star} \in(0, T)$ and performing integration by parts in space. As usual such a procedure can be justified via a Galerkin approximation. Hence the first desired result on $u$ in (3.20) follows on noting (3.3a). The second $u$ result in (3.20) then follows immediately from the first, (3.3a), the compact embedding $H^{d}(\Omega) \hookrightarrow C(\bar{\Omega})$ and (1.14b). Finally, the $v$ result in (3.20) follows from the $u$ result and the fact that $u|\nabla v| \in L^{2}\left(\Omega_{T}\right)$.

For any $\eta \in H^{1}\left(0, T ; H^{2}(\Omega)\right)$ we choose $\chi \equiv \pi^{h} \eta$ in (3.2b) and now analyse the subsequent terms. Firstly on noting (2.17), (2.13), (1.13) in time and (3.7), we have for all $\eta \in H^{1}\left(0, T ; H^{2}(\Omega)\right)$ that

$$
\begin{gathered}
\left|\int_{0}^{T}\left[\left(\frac{\partial V_{\varepsilon}}{\partial t}, \pi^{h} \eta\right)^{h}-\left(\frac{\partial V_{\varepsilon}}{\partial t}, \pi^{h} \eta\right)\right] \mathrm{d} t\right| \\
=\mid-\int_{0}^{T}\left(V_{\varepsilon}, \frac{\partial\left(\pi^{h} \eta\right)}{\partial t}\right)^{h} \mathrm{~d} t+\left(V_{\varepsilon}(\cdot, T), \pi^{h} \eta(\cdot, T)\right)^{h}-\left(V_{\varepsilon}(\cdot, 0), \pi^{h} \eta(\cdot, 0)\right)^{h} \\
\quad+\int_{0}^{T}\left(V_{\varepsilon}, \frac{\partial\left(\pi^{h} \eta\right)}{\partial t}\right) \mathrm{d} t-\left(V_{\varepsilon}(\cdot, T), \pi^{h} \eta(\cdot, T)\right)+\left(V_{\varepsilon}(\cdot, 0), \pi^{h} \eta(\cdot, 0)\right) \mid \\
\leqslant C h\left\|V_{\varepsilon}\right\|_{L^{\infty}\left(\Omega_{T}\right)}\|\eta\|_{H^{1}\left(0, T ; H^{2}(\Omega)\right)} \leqslant C h\|\eta\|_{H^{1}\left(0, T ; H^{2}(\Omega)\right)} .
\end{gathered}
$$


Similarly to (3.24), it follows from (2.13) and (3.7) that

$$
\begin{aligned}
\left|\int_{0}^{T}\left(\frac{\partial V_{\varepsilon}}{\partial t},\left(I-\pi^{h}\right) \eta\right) \mathrm{d} t\right| & \leqslant C h\left\|\frac{\partial V_{\varepsilon}}{\partial t}\right\|_{L^{2}\left(0, T ;\left(H^{1}(\Omega)\right)^{\prime}\right)}\|\eta\|_{L^{2}\left(0, T ; H^{2}(\Omega)\right)} \\
& \leqslant C h\|\eta\|_{L^{2}\left(0, T ; H^{2}(\Omega)\right)} \quad \forall \eta \in L^{2}\left(0, T ; H^{2}(\Omega)\right) .
\end{aligned}
$$

Combining (3.32), (3.33) and (3.5b) yields that

$$
\int_{0}^{T}\left(\frac{\partial V_{\varepsilon}}{\partial t}, \pi^{h} \eta\right)^{h} \mathrm{~d} t \rightarrow \int_{0}^{T}\left\langle\frac{\partial v}{\partial t}, \eta\right\rangle \mathrm{d} t \quad \text { as } h \rightarrow 0, \quad \forall \eta \in H^{1}\left(0, T ; H^{2}(\Omega)\right) .
$$

The desired result (3.22a) follows immediately from (3.6b), (3.4d), (3.21) and (3.3b). It follows from (2.20) and (3.7) that

$$
\left\|U_{\varepsilon}^{+} \Lambda_{\varepsilon}\left(V_{\varepsilon}^{+}\right) \nabla V_{\varepsilon}^{+}\right\|_{L^{2}\left(\Omega_{T}\right)} \leqslant C\left\|\left[U_{\varepsilon}^{+} \Lambda_{\varepsilon}\left(V_{\varepsilon}^{+}\right)\right]^{\frac{1}{2}} \nabla V_{\varepsilon}^{+}\right\|_{L^{2}\left(\Omega_{T}\right)} \leqslant C .
$$

In view of (3.35) and (2.13), we deduce for all $\eta \in L^{2}\left(0, T ; H^{2}(\Omega)\right)$ that

$$
\left|\int_{0}^{T}\left(U_{\varepsilon}^{+} \Lambda_{\varepsilon}\left(V_{\varepsilon}^{+}\right) \nabla V_{\varepsilon}^{+}, \nabla\left(I-\pi^{h}\right) \eta\right) \mathrm{d} t\right| \leqslant C h\|\eta\|_{L^{2}\left(0, T ; H^{2}(\Omega)\right)} .
$$

Furthermore, we have on noting (3.35) that there exists a limit $\underline{z} \in L^{2}\left(\Omega_{T}\right)$ such that for all $\eta \in$ $L^{2}\left(0, T ; H^{1}(\Omega)\right)$

$$
\int_{0}^{T}\left(U_{\varepsilon}^{+} \Lambda_{\varepsilon}\left(V_{\varepsilon}^{+}\right) \nabla V_{\varepsilon}^{+}, \nabla \eta\right) \mathrm{d} t \rightarrow \int_{0}^{T}(\underline{z}, \nabla \eta) \mathrm{d} t \quad \text { as } h \rightarrow 0 .
$$

In the remaining part of the proof we will establish that $\underline{z} \equiv \mathcal{H}_{\{u>0\}} u v \nabla v$. It follows from (2.20), (3.7), (1.13) and (3.4d) that for all $\eta \in L^{\infty}\left(0, T ; W^{1, \infty}(\Omega)\right)$

$$
\begin{aligned}
\mid \int_{\Omega_{T}}(1- & \left.\mathcal{H}_{\{u>0\}}\right) U_{\varepsilon}^{+} \Lambda_{\varepsilon}\left(V_{\varepsilon}^{+}\right) \nabla V_{\varepsilon}^{+} . \nabla \eta \mathrm{d} x \mathrm{~d} t \mid \\
& \leqslant C\left\|\left[U_{\varepsilon}^{+} \Lambda_{\varepsilon}\left(V_{\varepsilon}^{+}\right)\right]^{\frac{1}{2}} \nabla V_{\varepsilon}^{+}\right\|_{L^{2}\left(\Omega_{T}\right)}\left(\int_{\Omega_{T}}\left(1-\mathcal{H}_{\{u>0\}}\right)\left(U_{\varepsilon}^{+}-u\right)|\nabla \eta|^{2} \mathrm{~d} x \mathrm{~d} t\right)^{\frac{1}{2}} \\
& \leqslant C\left\|U_{\varepsilon}^{+}-u\right\|_{L^{2}\left(\Omega_{T}\right)}^{\frac{1}{2}}\|\eta\|_{L^{\infty}\left(0, T ; W^{1, \infty}(\Omega)\right)} \rightarrow 0 \text { as } h \rightarrow 0 .
\end{aligned}
$$

It follows from (3.37) and (3.38) that $\underline{z} \equiv 0$ a.e. on $\Omega_{T} \backslash\{u>0\}$. Next we note from (3.7), (3.4c,d) and $(3.6 \mathrm{~b}, \mathrm{c})$ that for any $\eta \in L^{\infty}\left(0, T ; W^{1, \infty}(\Omega)\right)$

$$
\begin{aligned}
\int_{\Omega_{T}}\left(U_{\varepsilon}^{+}\right)^{3} \nabla\left[\left(V_{\varepsilon}^{+}\right)^{2}\right] & \nabla \eta \mathrm{d} x \mathrm{~d} t \\
& =2 \int_{\Omega_{T}}\left[U_{\varepsilon}^{+}\left(U_{\varepsilon}^{+} V_{\varepsilon}^{+}\right) \nabla\left(U_{\varepsilon}^{+} V_{\varepsilon}^{+}\right)-\left(U_{\varepsilon}^{+} V_{\varepsilon}^{+}\right)^{2} \nabla U_{\varepsilon}^{+}\right] . \nabla \eta \mathrm{d} x \mathrm{~d} t \\
& \rightarrow 2 \int_{\Omega_{T}}\left[u(u v) \nabla(u v)-(u v)^{2} \nabla u\right] . \nabla \eta \mathrm{d} x \mathrm{~d} t \\
& =\int_{\Omega_{T}} u^{3} \nabla\left(v^{2}\right) . \nabla \eta \mathrm{d} x \mathrm{~d} t \quad \text { as } h \rightarrow 0 .
\end{aligned}
$$


Furthermore, we have on noting (3.7) that for any $\eta \in L^{\infty}\left(0, T ; W^{1, \infty}(\Omega)\right)$

$$
\begin{aligned}
& \left|\int_{\Omega_{T}}\left(U_{\varepsilon}^{+}\right)^{3}\left[\Lambda_{\varepsilon}\left(V_{\varepsilon}^{+}\right)-V_{\varepsilon}^{+} \mathcal{I}\right] \nabla V_{\varepsilon}^{+} \cdot \nabla \eta \mathrm{d} x \mathrm{~d} t\right| \\
& \quad \leqslant C\left\|\left(U_{\varepsilon}^{+}\right)^{\frac{1}{2}} \nabla V_{\varepsilon}^{+}\right\|_{L^{2}\left(\Omega_{T}\right)}\|\eta\|_{L^{\infty}\left(0, T ; W^{1, \infty}(\Omega)\right)}\left(\int_{\Omega_{T}}\left(U_{\varepsilon}^{+}\right)^{5}\left\|\Lambda_{\varepsilon}\left(V_{\varepsilon}^{+}\right)-V_{\varepsilon}^{+} \mathcal{I}\right\|^{2} \mathrm{~d} x \mathrm{~d} t\right)^{\frac{1}{2}} \\
& \quad \leqslant C\|\eta\|_{L^{\infty}\left(0, T ; W^{1, \infty}(\Omega)\right)}\left\|\left(U_{\varepsilon}^{+}\right)^{\frac{1}{2}}\right\| \Lambda_{\varepsilon}\left(V_{\varepsilon}^{+}\right)-V_{\varepsilon}^{+} \mathcal{I}\|\|_{L^{2}\left(\Omega_{T}\right)} .
\end{aligned}
$$

It follows from (3.7), (2.31), (2.12), (2.16), (2.39a,b), (2.30) and assumption (iii) that

$$
\begin{aligned}
& \left\|\left(U_{\varepsilon}^{+}\right)^{\frac{1}{2}}\right\| \Lambda_{\varepsilon}\left(V_{\varepsilon}^{+}\right)-V_{\varepsilon}^{+} \mathcal{I}\|\|_{L^{2}\left(\Omega_{T}\right)} \\
& \quad \leqslant\left\|\left(U_{\varepsilon}^{+}\right)^{\frac{1}{2}}\right\| \Lambda_{\varepsilon}\left(V_{\varepsilon}^{+}\right)-\pi^{h}\left[\lambda_{\varepsilon}\left(V_{\varepsilon}^{+}\right)\right] \mathcal{I}\|\|_{L^{2}\left(\Omega_{T}\right)}+\left\|\left(U_{\varepsilon}^{+}\right)^{\frac{1}{2}}\right\|\left(\pi^{h}\left[\lambda_{\varepsilon}\left(V_{\varepsilon}^{+}\right)\right]-V_{\varepsilon}^{+}\right) \mathcal{I}\|\|_{L^{2}\left(\Omega_{T}\right)} \\
& \quad \leqslant\left(\int_{0}^{T} \sum_{\kappa \in \mathcal{T}^{h}} h_{\kappa}^{2}\left|\left(U_{\varepsilon}^{+}\right)^{\frac{1}{2}} \nabla \pi^{h}\left[\lambda_{\varepsilon}\left(V_{\varepsilon}^{+}\right)\right]\right|_{0, \kappa}^{2} \mathrm{~d} t\right)^{\frac{1}{2}}+C\left\|\pi^{h}\left[\lambda_{\varepsilon}\left(V_{\varepsilon}^{+}\right)\right]-V_{\varepsilon}^{+}\right\|_{L^{2}\left(\Omega_{T}\right)} \\
& \quad \leqslant\left(\int_{0}^{T} \sum_{\kappa \in \mathcal{T}^{h}} h_{\kappa}^{2}\left|\left(U_{\varepsilon}^{+}\right)^{\frac{1}{2}} \nabla V_{\varepsilon}^{+}\right|_{0, \kappa}^{2} \mathrm{~d} t\right)^{\frac{1}{2}}+C\left\|\pi^{h}\left[\lambda_{\varepsilon}\left(V_{\varepsilon}^{+}\right)\right]-V_{\varepsilon}^{+}\right\|_{L^{2}\left(\Omega_{T}\right)} \\
& \quad \leqslant C h\left\|\left(U_{\varepsilon}^{+}\right)^{\frac{1}{2}} \nabla V_{\varepsilon}^{+}\right\|_{L^{2}\left(\Omega_{T}\right)}+C\left\|\pi^{h}\left[\lambda_{\varepsilon}\left(V_{\varepsilon}^{+}\right)-V_{\varepsilon}^{+}\right]\right\|_{L^{2}\left(\Omega_{T}\right)} \\
& \quad \leqslant C(h+\varepsilon) \quad \rightarrow 0 \text { as } h \rightarrow 0 .
\end{aligned}
$$

Hence we have on noting (3.4d), (3.35), (3.37), (3.40), (3.41) and (3.39) that for any $\eta \in$ $L^{\infty}\left(0, T ; W^{1, \infty}(\Omega)\right)$

$$
\begin{aligned}
2 \int_{\Omega_{T}} & u^{2} \underline{z} \cdot \nabla \eta \mathrm{d} x \mathrm{~d} t \leftarrow 2 \int_{\Omega_{T}}\left(U_{\varepsilon}^{+}\right)^{3} \Lambda_{\varepsilon}\left(V_{\varepsilon}^{+}\right) \nabla V_{\varepsilon}^{+} . \nabla \eta \mathrm{d} x \mathrm{~d} t \\
& =2 \int_{\Omega_{T}}\left(U_{\varepsilon}^{+}\right)^{3} V_{\varepsilon}^{+} \nabla V_{\varepsilon}^{+} \cdot \nabla \eta \mathrm{d} x \mathrm{~d} t+2 \int_{\Omega_{T}}\left(U_{\varepsilon}^{+}\right)^{3}\left[\Lambda_{\varepsilon}\left(V_{\varepsilon}^{+}\right)-V_{\varepsilon}^{+} \mathcal{I}\right] \nabla V_{\varepsilon}^{+} \cdot \nabla \eta \mathrm{d} x \mathrm{~d} t \\
& \rightarrow \int_{\Omega_{T}} u^{3} \nabla\left(v^{2}\right) . \nabla \eta \mathrm{d} x \mathrm{~d} t
\end{aligned}
$$

Therefore it follows from (3.42) that

$$
2 u^{2} \underline{z}=u^{3} \nabla\left(v^{2}\right) \text { a.e. on } \Omega_{T} \quad \Longrightarrow \quad \underline{z}=\frac{1}{2} u \nabla\left(v^{2}\right) \text { a.e. on }\{u>0\} \text {. }
$$

Combining (3.43), (3.36), (3.37) and (3.38), and noting (1.13) yields that

$$
\int_{0}^{T}\left(U_{\varepsilon}^{+} \Lambda_{\varepsilon}\left(V_{\varepsilon}^{+}\right) \nabla V_{\varepsilon}^{+}, \nabla\left(\pi^{h} \eta\right)\right) \mathrm{d} t \rightarrow \int_{\{u>0\}} u v \nabla v . \nabla \eta \mathrm{d} x \mathrm{~d} t \quad \forall \eta \in H^{1}\left(0, T ; H^{3}(\Omega)\right)
$$

and the desired result (3.22b). It follows from (3.2b), (1.4), (3.44), (3.34), (3.30), the denseness of $H^{1}\left(0, T ; H^{3}(\Omega)\right)$ in $L^{2}\left(0, T ; H^{1}(\Omega)\right)$ and (3.7) that (3.23b) holds. 
Finally, it follows from $(3.4 \mathrm{~b}, \mathrm{c}),(3.5 \mathrm{a}, \mathrm{b})$ and $(1.14 \mathrm{~b})$ that

$$
U_{\varepsilon} \rightarrow u \quad \text { in } \quad C\left([0, T] ; L^{2}(\Omega)\right) \quad \text { and } \quad V_{\varepsilon} \rightarrow v \quad \text { in } \quad C\left([0, T] ;\left(H^{1}(\Omega)\right)^{\prime}\right) .
$$

Furthermore, the assumptions $(i),(2.14)$ and (2.19) yield that

$$
U_{\varepsilon}^{0} \rightarrow u^{0} \quad \text { and } \quad V_{\varepsilon}^{0} \rightarrow v^{0} \quad \text { strongly in } \quad L^{2}(\Omega) .
$$

Combining (3.45) and (3.46) we obtain that $u(\cdot, 0)=u^{0}(\cdot), v(\cdot, 0)=v^{0}(\cdot)$.

REMARK 3.1 For initial data $u^{0}, v^{0} \in H^{2}(\Omega)$, it is convenient in practice to choose $U_{\varepsilon}^{0} \equiv \pi^{h} u^{0}$ and $V_{\varepsilon}^{0} \equiv \pi^{h} v^{0}$ for $d=2$. We note that all of the results in the above lemmas and theorems obviously remain true on noting (2.13). Also, in this case we do not require (2.19) for (2.60), (3.14) and (3.46) to hold. Hence we only need the quasi-uniformity assumption in order to obtain $(2.59 \mathrm{a}, \mathrm{b})$. However, we can replace this with far milder assumption that $\left\{\mathcal{T}^{h}\right\}_{h>0}$ is a regular partitioning at the expense of a minimum time step constraint as in [3]. Introducing $\mathcal{G}:\left(H^{1}(\Omega)\right)^{\prime} \rightarrow H^{1}(\Omega)$ and $\mathcal{G}^{h}: C(\bar{\Omega}) \rightarrow S^{h}$ such that

$$
\begin{array}{rlrl}
(\nabla \mathcal{G} z, \nabla \eta)+(\mathcal{G} z, \eta) & =\langle z, \eta\rangle & & \forall \eta \in H^{1}(\Omega), \\
\left(\nabla \mathcal{G}^{h} z, \nabla \chi\right)+\left(\mathcal{G}^{h} z, \chi\right) & =(z, \chi)^{h} & \forall \chi \in S^{h} .
\end{array}
$$

It is easily established from $\left\{\mathcal{T}^{h}\right\}_{h>0}$ being a regular partitioning, elliptic regularity, as $\Omega$ is convex polygonal if $d=2$, and (2.17) that

$$
\left\|\left(\mathcal{G}-\mathcal{G}^{h}\right) z^{h}\right\|_{1} \leqslant C h\left\|z^{h}\right\|_{0} \quad \forall z^{h} \in S^{h} .
$$

Then testing (2.11b) with $\chi \equiv \mathcal{G}^{h} \frac{\partial V_{\varepsilon}}{\partial t}$ we obtain, similarly to (2.59a), that

$$
\left\|\mathcal{G}^{h} \frac{\partial V_{\varepsilon}}{\partial t}\right\|_{L^{2}\left(0, T ; H^{1}(\Omega)\right)} \leqslant C
$$

on noting (2.60). Combining (3.48), (3.49) and noting the fourth bound in (2.54), (2.16) and (3.1a), it follows that

$$
\begin{aligned}
\left\|\mathcal{G} \frac{\partial V_{\varepsilon}}{\partial t}\right\|_{L^{2}\left(0, T ; H^{1}(\Omega)\right)} & \leqslant\left\|\left(\mathcal{G}-\mathcal{G}^{h}\right) \frac{\partial V_{\varepsilon}}{\partial t}\right\|_{L^{2}\left(0, T ; H^{1}(\Omega)\right)}+\left\|\mathcal{G}^{h} \frac{\partial V_{\varepsilon}}{\partial t}\right\|_{L^{2}\left(0, T ; H^{1}(\Omega)\right)} \\
& \leqslant C h\left\|\frac{\partial V_{\varepsilon}}{\partial t}\right\|_{L^{2}\left(\Omega_{T}\right)}+C \leqslant C\left(\tau_{\min }^{-\frac{1}{2}} h+1\right) \leqslant C,
\end{aligned}
$$

if the mild time step constraint $C h^{2} \leqslant \tau_{\min }:=\min _{n=1, \ldots, N} \tau_{n}$ is satisfied.

REMARK 3.2 If instead of $(1.2) b(\cdot, \cdot)$ was just degenerate in $s$, i.e. $b(r, s):=\sigma s$, then $(1.1 \mathrm{~b})$ would be a porous medium equation with a reaction term. A natural discretization of this would then be $(2.11 \mathrm{~b})$ with the second term on the left-hand side replaced by $\frac{1}{2} \sigma\left(\nabla \pi^{h}\left[\left(V_{\varepsilon}^{n}\right)^{2}\right], \nabla \chi\right)$, see e.g. [9]. This would suggest that a natural discretization of $(1.1 \mathrm{~b})$ with $b(\cdot, \cdot)$ defined by (1.2) would be

$$
\left(\frac{V_{\varepsilon}^{n}-V_{\varepsilon}^{n-1}}{\tau_{n}}, \chi\right)^{h}+\frac{1}{2} \sigma\left(U_{\varepsilon}^{n} \nabla \pi^{h}\left[\left(V_{\varepsilon}^{n}\right)^{2}\right], \nabla \chi\right)=\theta\left(g\left(U_{\varepsilon}^{n}, V_{\varepsilon}^{n-1}\right), \chi\right)^{h} \quad \forall \chi \in S^{h} .
$$


The approximation (3.51) has two advantages over (2.11b) in that it is a simple matter (a) to establish the existence and uniqueness of $V_{\varepsilon}^{n}$ satisfying (3.51), whereas the uniqueness of $V_{\varepsilon}^{n}$ satisfying (2.11b) is not guaranteed; (b) to construct and prove convergence of globally convergent iterative methods to solve the nonlinear system (3.51) for $V_{\varepsilon}^{n}$, whereas convergence of algorithms for solving (2.11b) is not guaranteed, see (4.2) below. However, for the approximation (3.51) it does not appear possible to prove a discrete analogue of (1.11), the sixth bound in (2.54), which plays such a key role in our convergence proof.

\section{Numerical results}

Before presenting some numerical results in both one and two space dimensions, we briefly state algorithms for solving the resulting system of algebraic equations for $\left\{U_{\varepsilon}^{n}, V_{\varepsilon}^{n}\right\}$ arising at each time level from the approximation $\left(\mathrm{P}_{\varepsilon}^{h, \tau}\right)$ in the model case $b(r, s):=\sigma r s$ and $g(r, s):=r s$. As (2.11a) is independent of $V_{\varepsilon}^{n}$, we first solve the resulting linear equation

$$
\left(\frac{U_{\varepsilon}^{n}-U_{\varepsilon}^{n-1}}{\tau_{n}}, \chi\right)^{h}+c\left(\nabla U_{\varepsilon}^{n}, \nabla \chi\right)+\left(U_{\varepsilon}^{n} V_{\varepsilon}^{n-1}, \chi\right)^{h}=0 \quad \forall \chi \in S^{h}
$$

to obtain $U_{\varepsilon}^{n}$. Then the nonlinear equation (2.11b) is solved for $V_{\varepsilon}^{n}$. In order to achieve this, we use the following iterative procedure: for $k \geqslant 1$ find $V_{\varepsilon}^{n, k} \in S^{h}$ such that

$$
\left(\frac{V_{\varepsilon}^{n, k}-V_{\varepsilon}^{n-1}}{\tau_{n}}, \chi\right)^{h}+\sigma\left(U_{\varepsilon}^{n} \Lambda_{\varepsilon}\left(V_{\varepsilon}^{n, k-1}\right) \nabla V_{\varepsilon}^{n, k}, \nabla \chi\right)=\theta\left(U_{\varepsilon}^{n} V_{\varepsilon}^{n-1}, \chi\right)^{h} \quad \forall \chi \in S^{h},
$$

where $V_{\varepsilon}^{n, 0} \equiv V_{\varepsilon}^{n-1}$. (4.2) is the natural extension of the iterative procedure proposed in [6] for solving a finite-element approximation of (1.8). As (4.2) is linear, existence of $V_{\varepsilon}^{n, k}$ follows from uniqueness; and this is easily established on noting (2.6a) and (2.39a). Hence the iteration (4.2) is well defined. We adopted the stopping criterion

$$
\left\|V_{\varepsilon}^{n, k}-V_{\varepsilon}^{n, k-1}\right\|_{0, \infty}<t o l
$$

with $t o l=10^{-8}$ and then set $V_{\varepsilon}^{n} \equiv V_{\varepsilon}^{n, k}$. Although we are unable to show convergence of this iteration, we observed good convergence properties in practice.

The linear systems (4.1) and (4.2), for each $k$, can be solved efficiently using a conjugate gradient algorithm. As the iteration in general took only a few steps to fulfil the stopping criterion, the analogue of (4.3) for successive iterates, we did not employ a preconditioner.

We note for later purposes that (4.2) can easily be adapted to handle diffusion coefficients of the form $b(r, s):=b_{1}(r) s$ with $b_{1}(r) \in C\left(\mathbf{R}_{\geqslant 0}\right)$ and $b_{1}(r) \geqslant 0$. In this case the term $\sigma\left(U_{\varepsilon}^{n} \Lambda_{\varepsilon}\left(V_{\varepsilon}^{n, k-1}\right) \nabla V_{\varepsilon}^{n, k}, \nabla \chi\right)$ in (4.2) is replaced by $\left(\pi^{h}\left[b_{1}\left(U_{\varepsilon}^{n}\right)\right] \Lambda_{\varepsilon}\left(V_{\varepsilon}^{n, k-1}\right) \nabla V_{\varepsilon}^{n, k}, \nabla \chi\right)$. In the simpler case that $b(r, s):=b_{1}(r)$, we solve the following linear system at each time level:

$$
\left(\frac{V_{\varepsilon}^{n}-V_{\varepsilon}^{n-1}}{\tau_{n}}, \chi\right)^{h}+\left(\pi^{h}\left[b_{1}\left(U_{\varepsilon}^{n}\right)\right] \nabla V_{\varepsilon}^{n}, \nabla \chi\right)=\theta\left(U_{\varepsilon}^{n} V_{\varepsilon}^{n-1}, \chi\right)^{h} \quad \forall \chi \in S^{h} .
$$

For the initial profiles we chose

$$
\begin{array}{ll}
\text { either } \quad(\text { i }) \quad u^{0}(x) \equiv u_{\max }^{0}, \quad v^{0}(x) \equiv v_{\max }^{0} \exp \left(-A|x|^{2}\right) \\
\text { or } \quad \text { (ii) } \quad u^{0}(x) \equiv u_{\max }^{0}, \quad v^{0}(x) \equiv v_{R}^{0}(|x|):=\frac{v_{\max }^{0}}{2}\left[1-\tanh \left(B\left(|x|-x_{0}\right)\right)\right] ;
\end{array}
$$



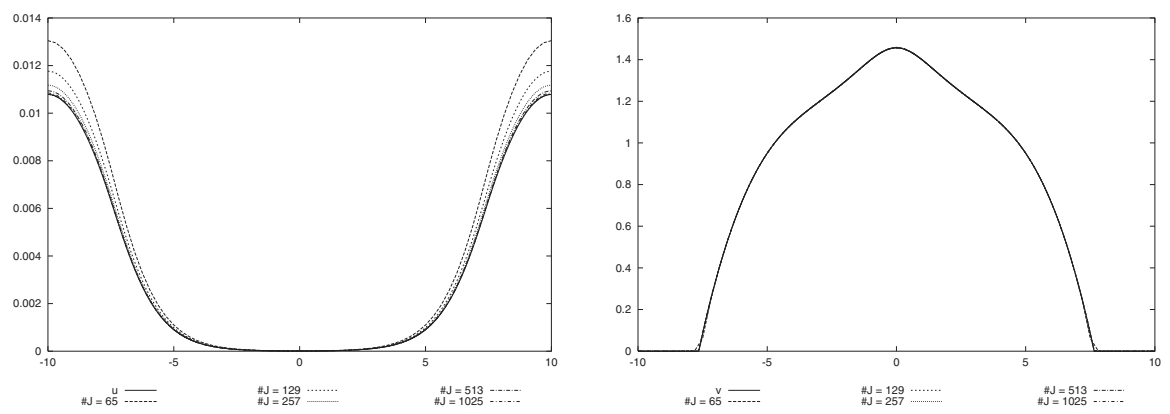

FIG. 1. $U_{\varepsilon}(x, 50)$ and $V_{\mathcal{E}}(x, 50)$ for $\mathcal{J}=2^{k}+1(k=6, \ldots, 10)$ and $\widehat{u}(x, 50), \widehat{v}(x, 50)$.

and set $U_{\varepsilon}^{0} \equiv u^{0}$ and $V_{\varepsilon}^{0} \equiv \pi^{h} v^{0}$ for simplicity as $v^{0} \in H^{2}(\Omega)$, see Remark 3.1.

\subsection{Numerical results for $d=1$}

We consider a uniform partitioning of $\Omega=(-L, L)$ with mesh points $p_{j}=-L+(j-1) h$, $j=1, \ldots, \mathcal{J}$, where $h=\frac{2 L}{\mathcal{J}-1}$. As no exact solution to $(\mathrm{P})$ is known, a comparison between the solutions of $\left(\mathrm{P}_{\varepsilon}^{h, \tau}\right)$ on a coarse mesh, $\left\{U_{\varepsilon}, V_{\varepsilon}\right\}$, with those on a fine mesh, $\{\widehat{u}, \widehat{v}\}$, was made. The data used in each experiment on the coarse meshes were $L=10, T=50, \tau_{n} \equiv \tau=1.25 h, \varepsilon=$ $1.25 \times 10^{-3} h$, where $\mathcal{J}=2^{k}+1(k=6, \ldots, 10), c=\sigma=\theta=1$ and $A=1, v_{\max }^{0}=u_{\max }^{0}=0.71$ for the initial profiles in (4.5a). The data were the same for the fine mesh except $\mathcal{J}=2^{13}+1$. The computed error bounds are given in the table below, where it appears that the $L^{\infty}\left(\Omega_{T}\right)$ error for both $u$ and $v$ is converging at the rate $O(h)$. A plot of the respective solutions can be seen in Fig. 1 .

\begin{tabular}{cccccc}
\hline & \multicolumn{5}{c}{$\mathcal{J}$} \\
\cline { 3 - 6 } & 65 & 129 & 257 & 513 & 1025 \\
\hline $\max _{n=1, \ldots, N}\left\|\pi^{h} \widehat{u}\left(\cdot, t_{n}\right)-U_{\varepsilon}^{n}(\cdot)\right\|_{0, \infty} \times 10^{3}$ & 56.28 & 29.56 & 15.00 & 7.380 & 3.473 \\
$\max _{n=1, \ldots, N}\left\|\pi^{h} \widehat{v}\left(\cdot, t_{n}\right)-V_{\varepsilon}^{n}(\cdot)\right\|_{0, \infty} \times 10^{3}$ & 75.50 & 39.21 & 19.83 & 9.745 & 4.584 \\
\hline
\end{tabular}

For the remainder of the results reported in this section we fixed $\tau_{n} \equiv \tau=10^{-3}, \varepsilon=10^{-5}, \mathcal{J}=$ $2^{10}+1$ and $c=\theta=1$. For $L=10$ and the same initial data as above we performed experiments to study the behaviour of the solutions. We observed that the computed solutions $U_{\varepsilon}(x, t)$ and $V_{\varepsilon}(x, t)$ approach steady states for $t$ sufficiently large; see Fig. 2, where we plot $U_{\varepsilon}\left(x, t_{n}\right)$ and $V_{\varepsilon}\left(x, t_{n}\right)$ for $t_{n}=0,10,100,200$. Note that $U_{\varepsilon}(x, t) \approx 0$ and $V_{\varepsilon}(x, t) \equiv \widehat{V}(x)$ for $t \geqslant 100$, which is due to the degeneracy of $b(\cdot, \cdot)$. This means in particular, that the $V_{\varepsilon}$ solution is 'frozen in' before a stable profile is established, so that no travelling wave is created.

For larger choices of $L$ and $T$, however, one can observe travelling wave solutions. For $L=$ 150 and keeping all other parameters fixed, we include the plots of $U_{\varepsilon}\left(x, t_{n}\right)$ and $V_{\varepsilon}\left(x, t_{n}\right)$ for $t_{n}=0,400, \ldots, 4000$; see Fig. 3, where one can clearly see the effect of the degenerate diffusion coefficient $b(\cdot, \cdot)$. At sufficiently late times the established 'spike' in $V_{\varepsilon}$ can not spread out any further, since $U_{\varepsilon}$ is practically zero in this area. In areas where the nutrient supply is still sufficient, however, the growth of the bacteria colony continues, creating two sharp fronts. Of course $b(r, s) \equiv$ 

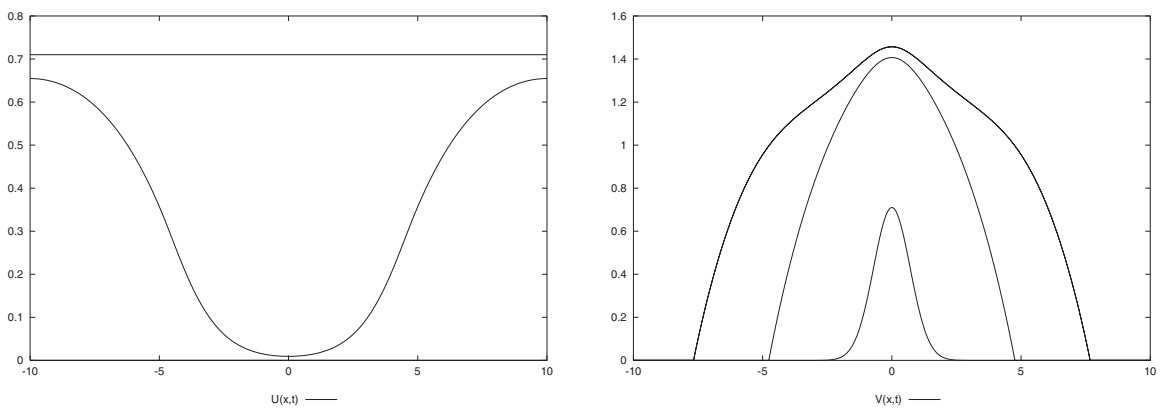

FIG. 2. $U_{\varepsilon}\left(x, t_{n}\right)$ and $V_{\varepsilon}\left(x, t_{n}\right)$ for $t_{n}=0,10,100,200$.
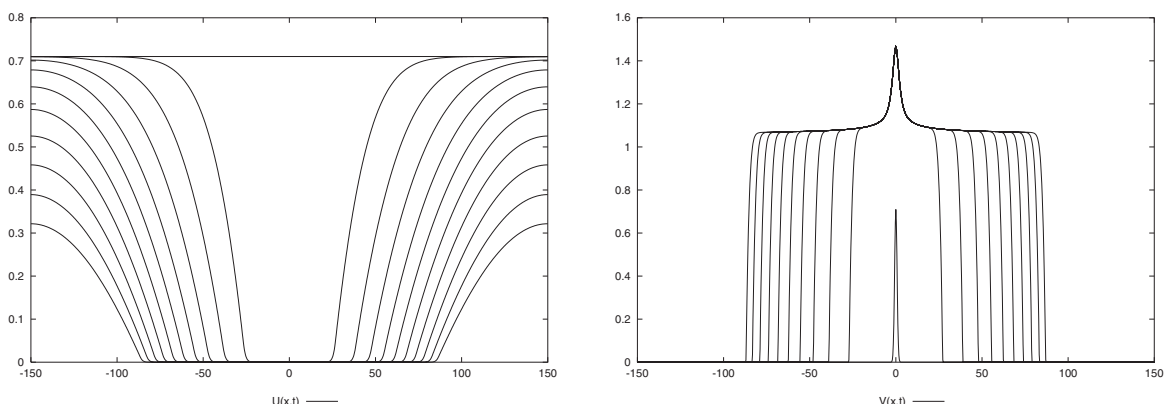

FIG. 3. $U_{\varepsilon}\left(x, t_{n}\right)$ and $V_{\varepsilon}\left(x, t_{n}\right)$ for $t_{n}=0,400, \ldots, 4000$.
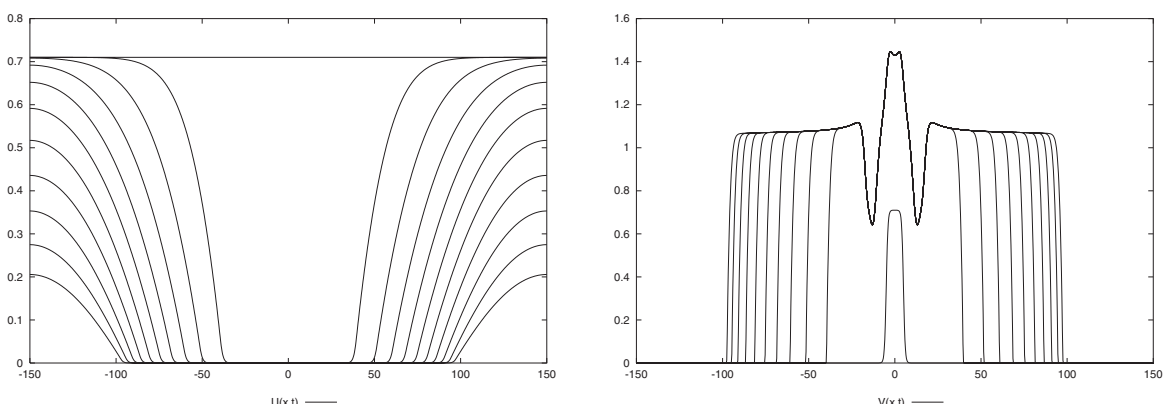

FIG. 4. $U_{\varepsilon}\left(x, t_{n}\right)$ and $V_{\varepsilon}\left(x, t_{n}\right)$ for $t_{n}=0,400, \ldots, 4000$.

$r s$ is only 'non-zero' in the small interfacial region behind the sharp fronts in $V_{\varepsilon}$, see also Fig. 5. It is worth mentioning that the shape of the initial profiles has a considerable effect on the evolution. This is underlined by the plots in Fig. 4, where we chose $B=1, x_{0}=5$ and $v_{\max }^{0}=u_{\max }^{0}=0.71$ in (4.5b) and kept the remaining parameters as before. Again one can observe the impact of the double degeneracy of $b(\cdot, \cdot)$. For the benefit of the reader we also include plots of the diffusion coefficient $b(r, s)$. As mentioned before it is effective in a very small region only; as can be seen in Fig. 5, where it is plotted twice with different scales for the vertical axis. Note that the speed of the sharp $V_{\varepsilon}$ fronts decreases as the diffusion coefficient decreases.

Furthermore we investigated the effect of different diffusion coefficients $b(\cdot, \cdot)$. Firstly, we 

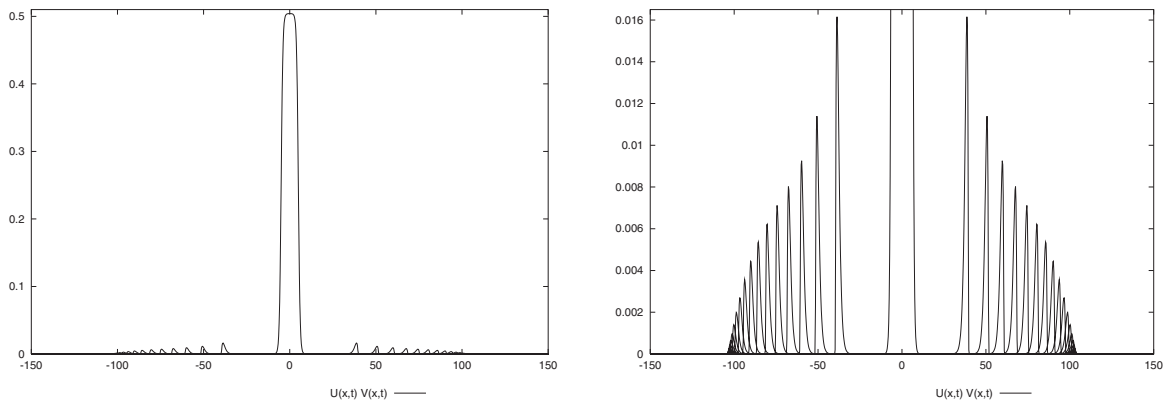

FIG. 5. $b\left(U_{\varepsilon}\left(x, t_{n}\right), V_{\varepsilon}\left(x, t_{n}\right)\right)$ for $t_{n}=0,400, \ldots, 4000$.
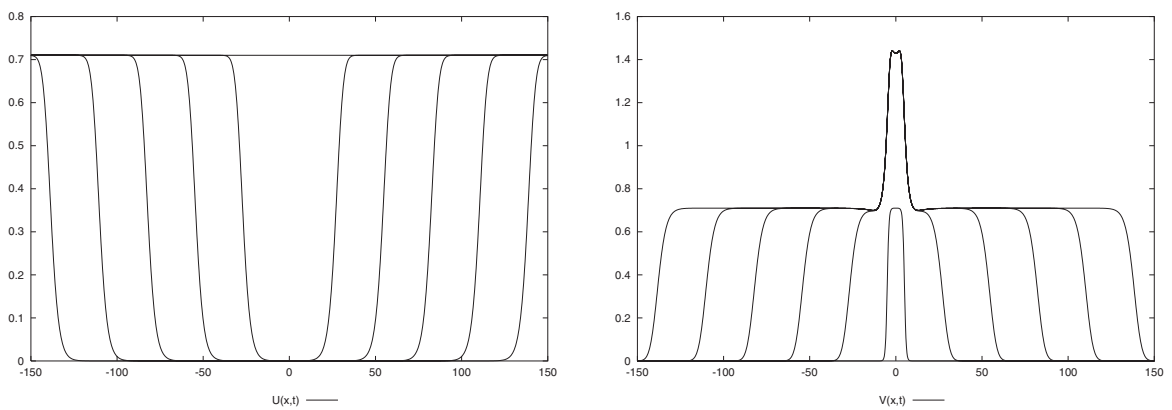

FIG. 6. $U_{\mathcal{\varepsilon}}\left(x, t_{n}\right)$ and $V_{\varepsilon}\left(x, t_{n}\right)$ for $t_{n}=0,20, \ldots, 100$ with $b(r, s):=r$.
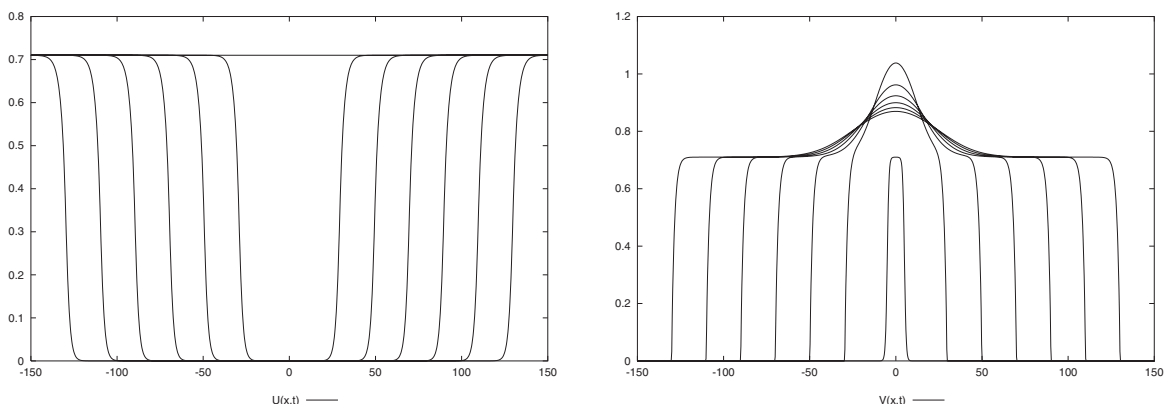

FIG. 7. $U_{\varepsilon}\left(x, t_{n}\right)$ and $V_{\varepsilon}\left(x, t_{n}\right)$ for $t_{n}=0,50, \ldots, 300$ with $b(r, s):=s$

performed an experiment for the degenerate case $b(r, s):=r$, see Fig. 6, while keeping the other parameters fixed throughout: $L=150$, and $B=1, x_{0}=5, v_{\max }^{0}=u_{\max }^{0}=0.71$ in (4.5b). In the second experiment we chose $b(r, s):=s$, see Fig. 7. Finally, we performed an experiment for the non-degenerate case $b(r, s):=1$, see Fig. 8. Note that the 'spike' in $V_{\varepsilon}$ continues to spread out in the last two cases. Moreover, sharp fronts in $V_{\varepsilon}$ can only be observed for the cases where $b(r, s)$ is degenerate in $s$, which is to be expected as (1.1b) is then similar to a porous medium equation with a reaction term; see Remark 3.2. For all four diffusion coefficients the solutions eventually reach a state that is (almost) stable with respect to time. In the first two cases the solutions remain constant due to the type of the degeneracy of $b(\cdot, \cdot)$ and the fact that $U_{\varepsilon}(x, t) \approx 0$ for $t$ sufficiently large. 

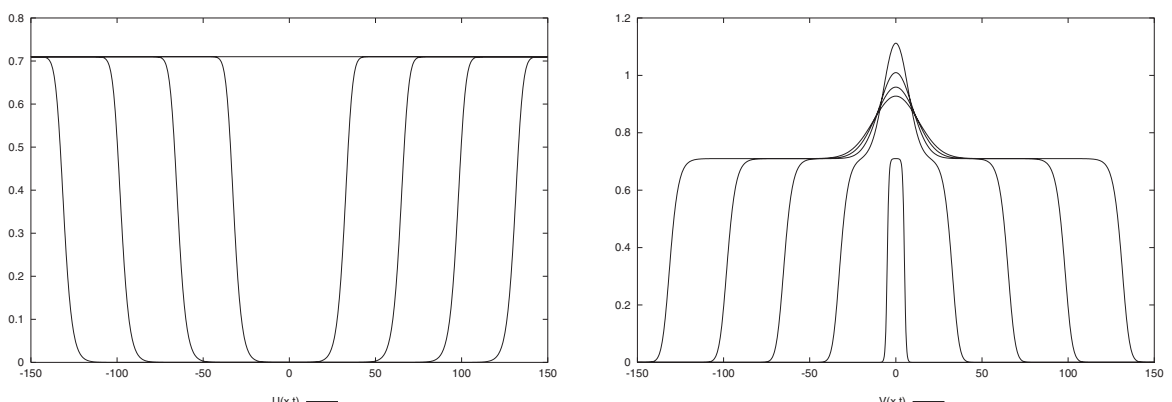

FIG. 8. $U_{\varepsilon}\left(x, t_{n}\right)$ and $V_{\varepsilon}\left(x, t_{n}\right)$ for $t_{n}=0,20, \ldots, 80$ with $b(r, s)=1$.
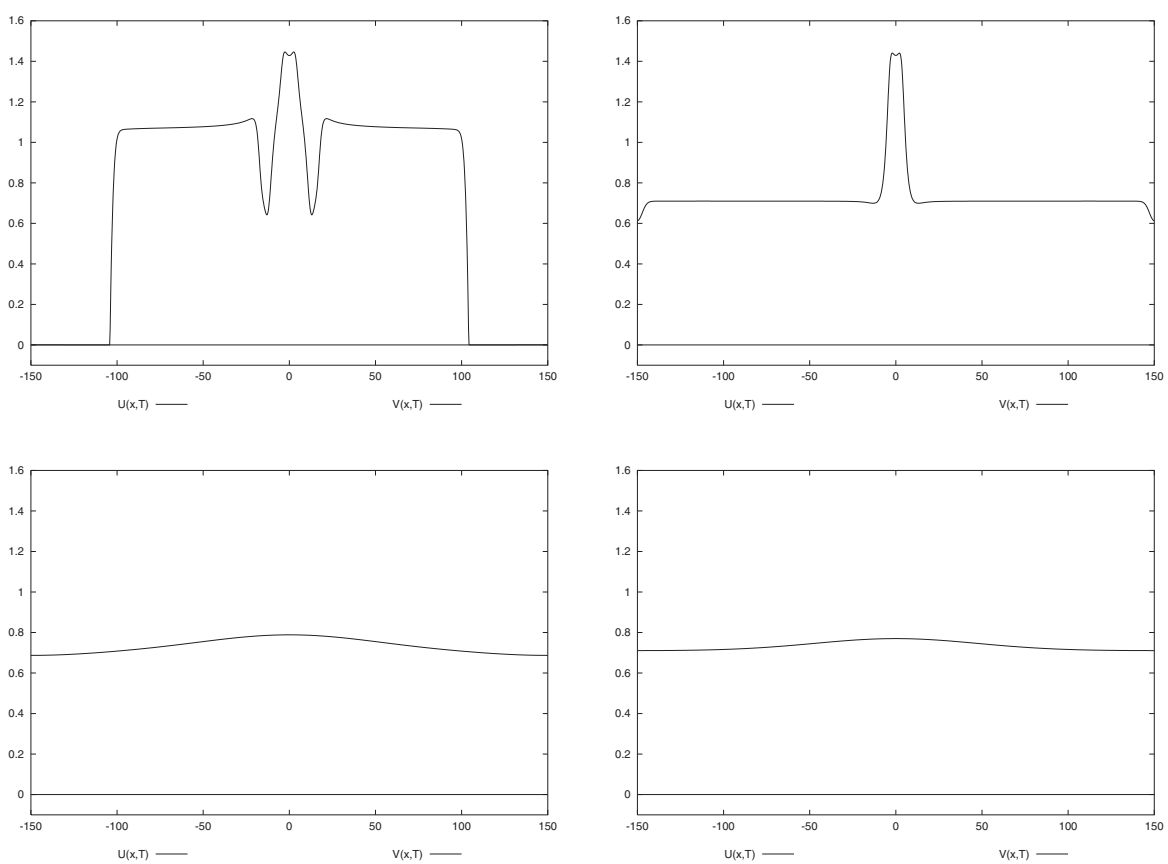

FIG. 9. The solutions for the four different diffusion coefficients at $T=10000$.

In the last two cases $V_{\varepsilon}$ keeps spreading very slowly. We give the solutions at $T=10000$ for all considered diffusion coefficients in Fig. 9.

\subsection{Numerical results for $d=2$}

Finally, we present numerical experiments in two space dimensions with $\Omega=(-L, L) \times(-L, L)$. We took a uniform mesh of squares of length $h=2 L /\left[(\mathcal{J})^{\frac{1}{2}}-1\right]$, each of which was divided into two triangles by its north east diagonal.

As for $d=1$ we performed experiments for the initial profiles in (4.5b). In particular we chose $B=1, x_{0}=5, v_{\max }^{0}=0.71, u_{\max }^{0}=0.35$ and $L=50, T=1200, c=\sigma=\theta=1, \tau_{n} \equiv \tau=$ 

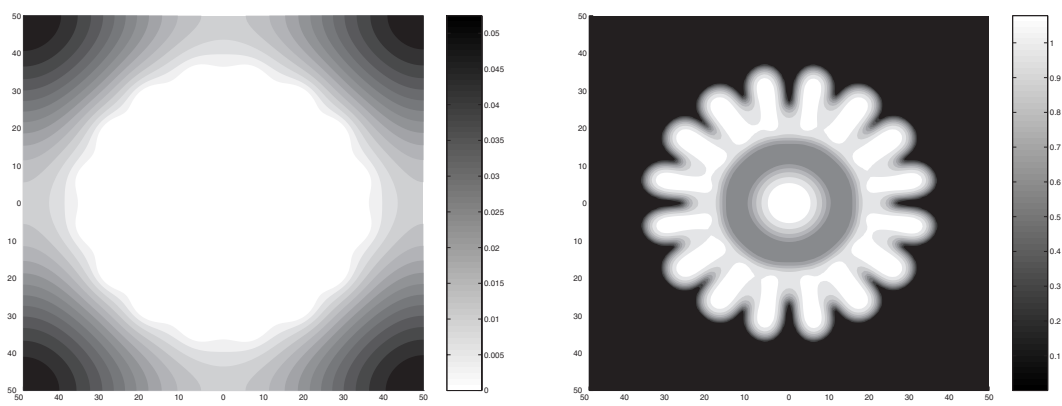

FIG. 10. Contour plots of $U_{\varepsilon}(x, 1200)$ and $V_{\varepsilon}(x, 1200)$ for $\mathcal{J}=\left(2^{7}+1\right)^{2}$.
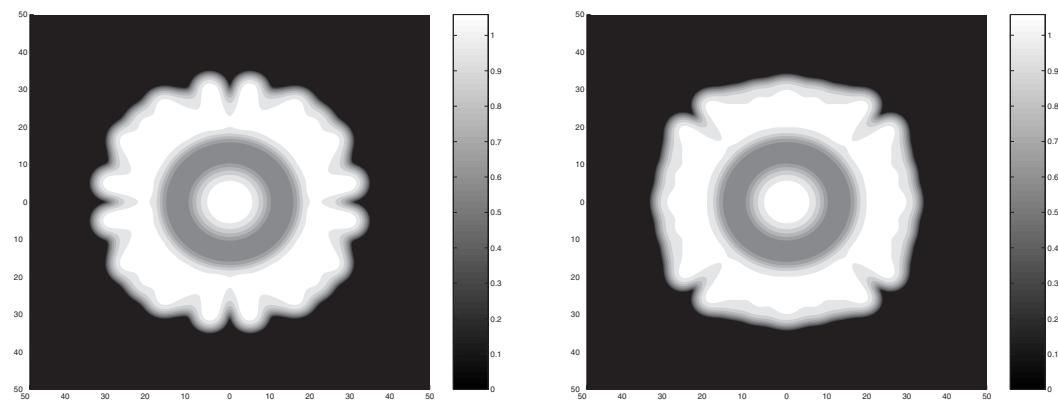

FIG. 11. Contour plots of $V_{\varepsilon}(x, 1200)$ for $\mathcal{J}=\left(2^{k}+1\right)^{2}(k=8,9)$.
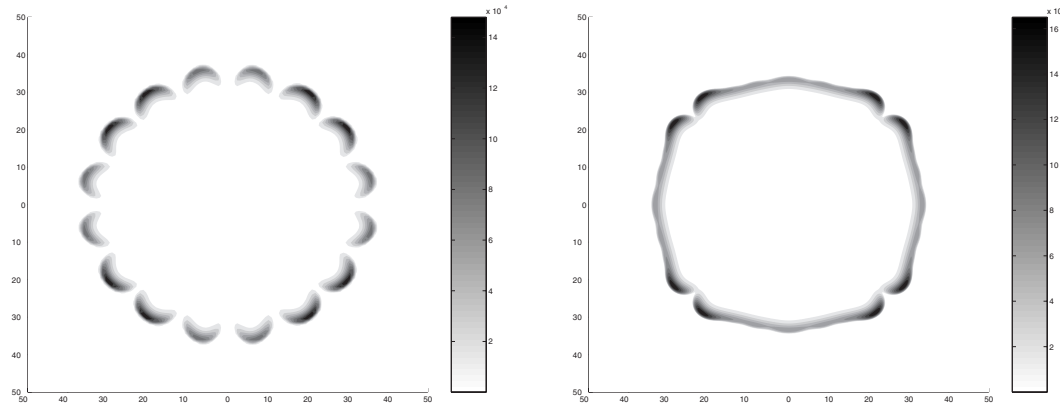

FIG. 12. Contour plots of $b\left(U_{\varepsilon}(x, 1200), V_{\varepsilon}(x, 1200)\right)$ for $\mathcal{J}=\left(2^{k}+1\right)^{2}(k=7,9)$

$5.12 \times 10^{-2} h, \varepsilon=5.12 \times 10^{-5} h$, where $\mathcal{J}=\left(2^{7}+1\right)^{2}$, for the remaining parameters. As can be seen from the contour plot of $V_{\varepsilon}$ in Fig. 10, the double degeneracy of the diffusion coefficient $b(\cdot, \cdot)$ leads to a fingering effect. This front instability conforms with numerical results reported in [7] and [5].

In order to validate these last results we performed experiments with the same parameters, but with finer mesh sizes $h$. In particular we chose $\mathcal{J}=\left(2^{k}+1\right)^{2}(k=8,9)$. One can observe from the plots in Fig. 11 that the finer the mesh, the less the solution $V_{\varepsilon}$ deviates from the radial symmetry of the initial profile $v^{0}$. Hence the fingering effect in Fig. 10 is due to mesh effects. In Fig. 12 we plot the regions where $b(r, s)$ is 'non-zero' for $\mathcal{J}=\left(2^{k}+1\right)^{2}(k=7,9)$ at $T=1200$. Note that 

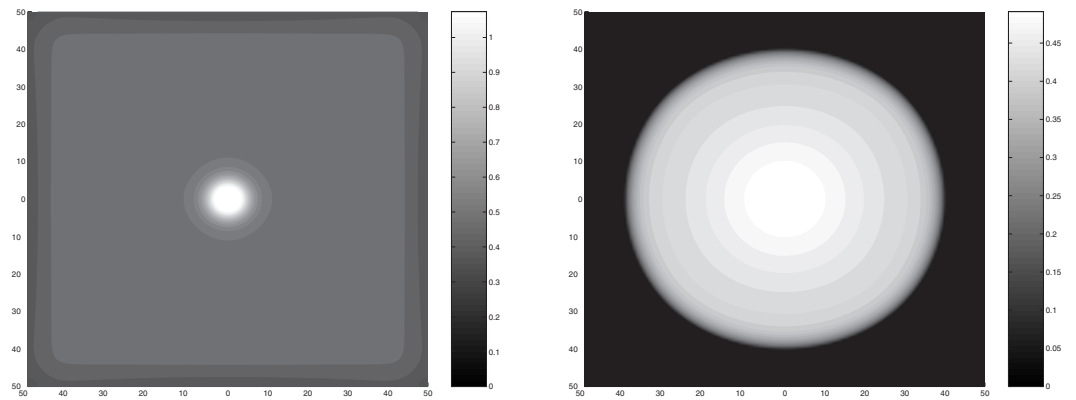

FIG. 13. Contour plots of $V_{\varepsilon}(x, 160)$ for $b(r, s):=r$ and $b(r, s):=s$, respectively, with $\mathcal{J}=\left(2^{7}+1\right)^{2}$.
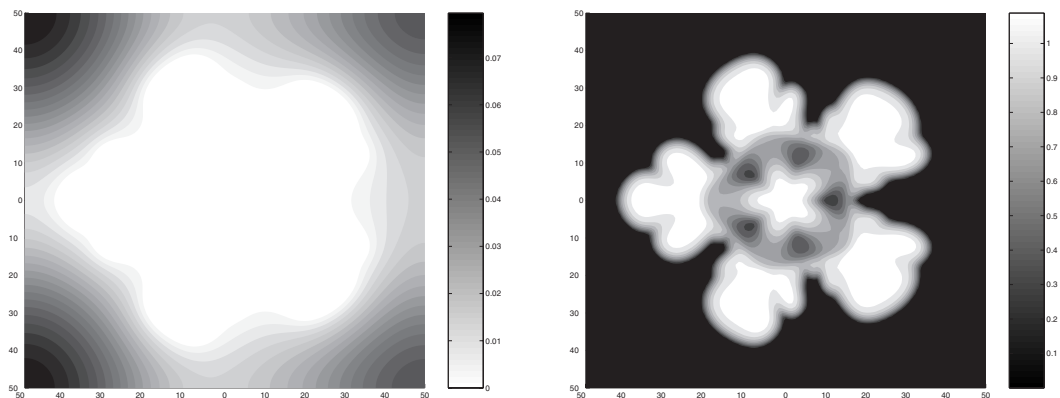

FIG. 14. Contour plots of $U_{\varepsilon}(x, 1000)$ and $V_{\varepsilon}(x, 1000)$ for $\mathcal{J}=\left(2^{7}+1\right)^{2}$.

the maximum value is approximately $1.6 \times 10^{-3}$ at this time and hence that the solutions in Figs 10 and 11 are close to steady states.

Furthermore, we again compared the behaviour of the solutions for different diffusion coefficients $b(\cdot, \cdot)$. It turned out that only the case $b(r, s):=\sigma r s$ produces 'interesting' results as in Fig. 10, where $\sigma=1$. Here we present experiments for the two degenerate cases $b(r, s):=r$ and $b(r, s):=s$ for the choice of $\mathcal{J}=\left(2^{7}+1\right)^{2}$. As the solutions advance with very different speeds compared to the double degeneracy case, we had to adjust the time scales accordingly. The results for the same initial data and parameters as before for $b(r, s):=r$ and $b(r, s):=s$ at $T=160$ can be seen in Fig. 13. Note that $V_{\varepsilon}$ has reached a steady state for $b(r, s):=r$ at this time. We did not include a figure for the non-degenerate case $b(r, s):=1$, as the contours look very similar to those in the plot on the right-hand side of Fig. 13.

Finally, we performed experiments where the initial data $v^{0}$ is not radially symmetric. Using polar coordinates, $(\rho, \phi)$, we chose an initial profile

$$
v_{\text {pert }}^{0}(\rho, \phi) \equiv v_{R}^{0}\left(\rho\left[1+\delta_{1} \cos (3 \phi)+\delta_{2} \cos (5 \phi)\right]\right),
$$

where $v_{R}^{0}$ is given by (4.5b), and $\delta_{1}:=0.05, \delta_{2}:=0.1$ are small perturbations to the radial symmetry of $v^{0}$. Keeping all other parameters as before, with $v^{0}$ replaced by (4.6), we plot the solutions for $b(r, s):=r s$ at $T=1000$ for $\mathcal{J}=\left(2^{k}+1\right)^{2}(k=7,8,9)$, see Figs 14 and 15 . The regions where the diffusion coefficient is effective at $T=1000$ is plotted in Fig. 16. Observe that the maximum value is approximately $2.0 \times 10^{-3}$ at this time and hence that the solutions in Figs 14 and 15 are 

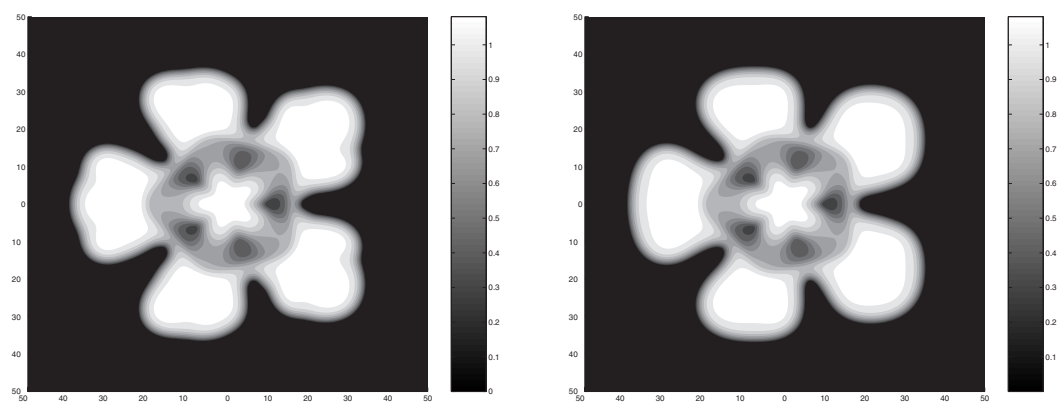

FIG. 15. Contour plots of $V_{\varepsilon}(x, 1000)$ for $\mathcal{J}=\left(2^{k}+1\right)^{2}(k=8,9)$.

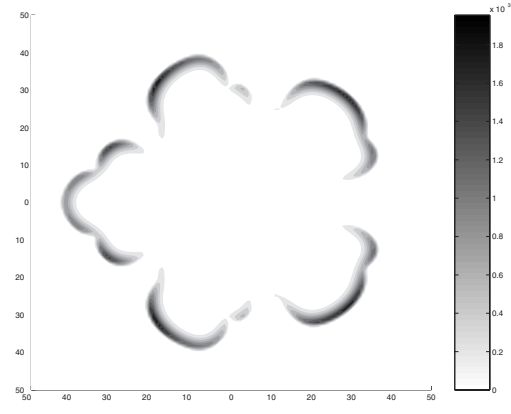

FIG. 16. Contour plot of $b\left(U_{\varepsilon}(x, 1000), V_{\varepsilon}(x, 1000)\right)$ for $\mathcal{J}=\left(2^{7}+1\right)^{2}$.
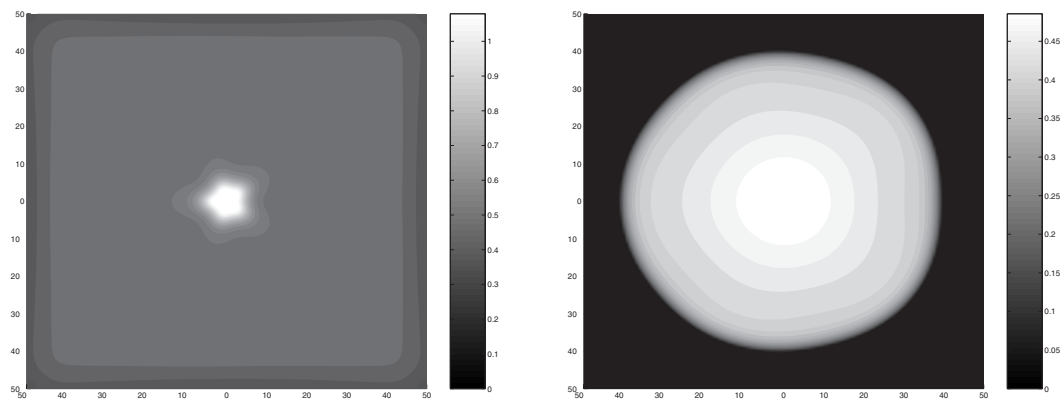

FIG. 17. Contour plots of $V_{\varepsilon}(x, 160)$ for $b(r, s):=r$ and $b(r, s):=s$, respectively, with $\mathcal{J}=\left(2^{7}+1\right)^{2}$.

close to steady states. For this doubly degenerate diffusion we see that a small perturbation in the initial data leads to a significant change in the evolution.

Once again one observes very different behaviour for the diffusion coefficients $b(r, s):=r$ and $b(r, s):=s$; see Fig. 17. In the former case the effect of the perturbation to the initial data is 'frozen in' about the origin as $U_{\varepsilon}$ approaches zero there in a short period of time, whereas the front is smeared; similarly to the case $d=1$, see Figs 6 and 9. In the latter case the perturbation of the initial data has negligible effect as the plots on the right-hand sides of Figs 13 and 17 are practically identical, as to be expected; see Figs 7 and 9 for $d=1$. 


\section{Acknowledgements}

R. N. was partially supported by the EPSRC, and by the DAAD through a Doktorandenstipendium.

\section{REFERENCES}

1. Adams, R. A. \& Fournier, J. Cone conditions and properties of Sobolev spaces. J. Math. Anal. Appl. 61, (1977) 713-734.

2. Barrett, J. W. \& Blowey, J. F. Finite element approximation of a degenerate Allen-Cahn/CahnHilliard system. SIAM J. Numer. Anal. 39, (2001) 1598-1624.

3. Barrett, J. W., Blowey, J. F., \& Garcke, H. Finite element approximation of a fourth order nonlinear degenerate parabolic equation. Numer. Math. 80, (1998) 525-556.

4. Deckelnick, K., Elliott, C. M., \& Styles, V. Numerical diffusion induced grain boundary motion. Interfaces and Free Boundaries 3, (2001) 393-414.

5. Golding, I., Kozlovsky, Y., Cohen, I., \& Ben-Jacob, E. Studies of bacterial branching growth using reaction-diffusion models for colonial development. Physica A 1998, (260) 510-554.

6. GRÜN, G. \& RUMPF, M. Nonnegativity preserving numerical schemes for the thin film equation. Numer. Math. 87, (2000) 113-152.

7. Kawasaki, K., Mochizuki, A., Matsushita, M., Umeda, T., \& Shigesada, N. Modelling spatiotemporal patterns generated by bacillus subtilis. J. Theor. Biol. 188, (1997) 177-185.

8. Mimura, M., Sakaguchi, H., \& Matsushita, M. Reaction-diffusion modelling of bacterial colony patterns. Physica A 282, (2000) 283-303.

9. Nochetto, R. H. \& Verdi, C. Approximation of degenerate parabolic problems using numerical integration. SIAM J. Numer. Anal. 25, (1988) 784-814.

10. OrtegA, J. M. \& Rheinboldt, W. C. Iterative Solution of Nonlinear Equations in Several Variables. Academic Press, New York (1970).

11. Renardy, M. \& Rogers, R. C. An Introduction to Partial Differential Equations. Springer, New York (1992).

12. Schmidt, A. \& Siebert, K. G. ALBERT: An adaptive hierarchical finite element toolbox. Manual, Preprint 06/2000, Freiburg, (2000).

13. Simon, J. Compact sets in the space $L^{p}(0, T ; B)$. Ann. Math. Pura. Appl. 146, (1981) 65-96.

14. ZhORNITSKAYA, L. \& BertozZI, A. L. Positivity preserving numerical schemes for lubrication-type equations. SIAM J. Numer. Anal. 37, (2000) 523-555. 